\title{
Total Synthesis of (-)-Crambidine and Definition of the Relative Configuration of Its Unique Tetracyclic Guanidinium Core
}

\author{
Larry E. Overman* and Young Ho Rhee \\ Department of Chemistry, 516 Rowland Hall, University of California, Irvine CA 92697-2025
}

\section{Supporting Information}

Experimental procedure for the preparation of $\mathbf{5 a}, \mathbf{5 b}, \mathbf{1 6}, \mathbf{1 8}, \mathbf{1 9}, \mathbf{2 1}, \mathbf{2 2}, \mathbf{2 3}$, 24, 25a, 25b, 26, 27; copies of ${ }^{1} \mathrm{H}$ and ${ }^{13} \mathrm{C}$ NMR spectra for new compounds and synthetic crambidine $\mathbf{4 a}$ and $\mathbf{4 b}$; tabulated characterization data for $\mathbf{6}$.

Tetracyclic acid 6. A solution of allyl ester $15(27 \mathrm{mg}, 0.034 \mathrm{mmol})$, tetrakis(triphenylphosphine)palladium (17 $\mathrm{mg}, 0.015 \mathrm{mmol})$, and morpholine (13 $\mu \mathrm{L}, \mathrm{mmol})$ was maintained in $\mathrm{MeCN}(0.34 \mathrm{~mL}$, degassed with Ar) at $\mathrm{rt}$ for $2 \mathrm{~h}$ under Ar. The reaction mixture was diluted with $\mathrm{MeCN}(10 \mathrm{~mL})$, filtered through a pad of Celite and concentrated. The residual oil was partitioned between $\mathrm{CHCl}_{3}(30 \mathrm{~mL})$ and $1 \mathrm{~N} \mathrm{HCl}(7 \mathrm{~mL})$. The organic layer was washed with $1 \mathrm{~N} \mathrm{HCl}(5 \mathrm{~mL})$ and brine $(2 \times 5 \mathrm{~mL})$, dried over anhydrous $\mathrm{Na}_{2} \mathrm{SO}_{4}$ and concentrated. The residual oil was purified by reverse phase HPLC (Phenomenex C-18, $10 \mathrm{ml} / \mathrm{min}, 10 \% \mathrm{MeCN}: 0.1 \% \mathrm{HCl}$ in $\mathrm{H}_{2} \mathrm{O}$ to $90 \% \mathrm{MeCN}: 0.1 \% \mathrm{HCl}$ in $\mathrm{H}_{2} \mathrm{O}$ over $20 \mathrm{~min}$, tR $=21.2 \mathrm{~min})$ to give 6 as clear oil $(19 \mathrm{mg}, 0.027 \mathrm{mmol}, 75 \%$ yield $):{ }^{1} \mathrm{H} \mathrm{NMR}(500 \mathrm{MHz}$, $\left.\mathrm{CD}_{3} \mathrm{OD}\right): \delta 5.76(\operatorname{app} \mathrm{t}, \mathrm{J}=9.1 \mathrm{~Hz}, 1 \mathrm{H}), 5.55($ br d J $=11 \mathrm{~Hz}, 1 \mathrm{H}), 4.84(\mathrm{~m}, 1 \mathrm{H}), 4.51-4.59$ $(\mathrm{m}, 1 \mathrm{H}), 4.37(\mathrm{t}, \mathrm{J}=6.6 \mathrm{~Hz}, 2 \mathrm{H}), 3.77-3.70(\mathrm{~m}, 1 \mathrm{H}), 3.63(\mathrm{dd}, \mathrm{J}=19,8 \mathrm{~Hz}, 1 \mathrm{H}), 3.60-3.50$ (m, 1H), 3.30-3.09 (m, 2H), $2.85(\mathrm{dd}, \mathrm{J}=13.4,3.6 \mathrm{~Hz}, 1 \mathrm{H}), 2.75-2.70(\mathrm{~m}, 1 \mathrm{H}), 2.64$ (app t, $\mathrm{J}=13 \mathrm{~Hz}, 1 \mathrm{H}), 2.48(\operatorname{app} \mathrm{t}, \mathrm{J}=14 \mathrm{~Hz}, 1 \mathrm{H}), 2.27(\mathrm{t}, \mathrm{J}=7.4 \mathrm{~Hz}, 2 \mathrm{H}), 2.28-2.20(\mathrm{~m}, 1 \mathrm{H})$, 2.16-2.09 (m, 1H), 2.08-2.02 (m, 1H), 1.92-1.72 (m, 4H), $1.70(\operatorname{app~t,~J~=~} 13 \mathrm{~Hz}, 1 \mathrm{H}), 1.54-$ $1.60(\mathrm{~m}, 3 \mathrm{H}), 1.52-1.47(\mathrm{~m}, 2 \mathrm{H}), 1.45-1.40(\mathrm{~m}, 2 \mathrm{H}), 1.38-1.28(\mathrm{~m}, 22 \mathrm{H}), 1.15(\mathrm{~d}, \mathrm{~J}=6.2$ $\mathrm{Hz}, 3 \mathrm{H}), 0.83(\mathrm{t}, \mathrm{J}=7.3 \mathrm{~Hz}, 3 \mathrm{H}) ;{ }^{13} \mathrm{C} \mathrm{NMR}\left(125 \mathrm{MHz}, \mathrm{CD}_{3} \mathrm{OD}\right): \delta 182.0,177.8,167.5$, $164.7,151.8,133.8,131.6,114.7,87.0,72.7,68.3,67.6,61.0,39.7,38.7,37.7,35.7,35.1$, $34.6,30.88,30.87,30.83,30.82,30.78,30.73,30.56,30.49,30.38,29.8,27.4,26.2$, 25.7, 25.0, 23.8, 10.7; IR (neat): 3246, 2926, 2856, 1722, 1629, 1583, 1297, 1258, 1235, 1127. $[\alpha]_{589}^{27}-19.0,[\alpha]_{577}^{27}-22.4,[\alpha]_{546}^{27}-31.5,[\alpha]^{27}{ }_{435}-105(c 0.10, \mathrm{MeOH}),[\alpha]_{405}^{27}-129(c$ 0.29, $\mathrm{MeOH}$ ); HRMS (ESI ${ }^{+}$) m/z 696.4939 (696.4928 calculated for $\mathrm{C}_{38} \mathrm{H}_{62} \mathrm{~N}_{3} \mathrm{O}_{6}\left[\mathrm{M}^{+}\right]$).

Tetracyclic acetate 16. A solution of alcohol $15(22 \mathrm{mg}, 0.030 \mathrm{mmol})$, pyridine $(0.3 \mathrm{~mL})$, and acetic anhydride $(0.2 \mathrm{~mL})$ was maintained at $\mathrm{rt}$ for $2 \mathrm{~h}$. The product was directly purified by reverse-phase preparative HPLC (Phenomenex C-18, 10 ml $/ \mathrm{min}, 10 \%$ $\mathrm{MeCN}: 0.1 \% \mathrm{HCl}$ in $\mathrm{H}_{2} \mathrm{O}$ to $90 \% \mathrm{MeCN}: 0.1 \% \mathrm{HCl}$ in $\mathrm{H}_{2} \mathrm{O}$ over $20 \mathrm{~min}$, $\mathrm{tR}=24 \mathrm{~min}$ ) to give 16 as a clear oil (17 mg, $0.022 \mathrm{mmol}, 70 \%$ yield): ${ }^{1} \mathrm{H}$ NMR (500 $\mathrm{MHz}, \mathrm{CDCl}_{3}$ ) 
$\delta 12.7$ (br s, 1H), 5.87-5.95 (m, 1H), $5.68(\mathrm{~m}, 1 \mathrm{H}), 5.52(\operatorname{app~d}, \mathrm{J}=10.9 \mathrm{~Hz}, 1 \mathrm{H}), 5.32(\mathrm{dq}$, $\mathrm{J}=17.2,1.5 \mathrm{~Hz}, 1 \mathrm{H}), 5.27(\mathrm{dq}, \mathrm{J}=10.4,1.4 \mathrm{~Hz}, 1 \mathrm{H}), 5.21$ (br s, 1H), $4.82(\mathrm{~m}, 2 \mathrm{H}), 4.57$ $(\operatorname{app~d}, \mathrm{J}=5.7 \mathrm{~Hz}, 2 \mathrm{H}), 4.32(\mathrm{br} \mathrm{s}, 2 \mathrm{H}), 3.65(\mathrm{~m}, 2 \mathrm{H}), 3.38(\mathrm{~m}, 1 \mathrm{H}), 3.12(\mathrm{~m}, 2 \mathrm{H}), 2.72(\mathrm{~m}$, $1 \mathrm{H}), 2.64(\mathrm{~m}, 1 \mathrm{H}), 2.34-2.28(\mathrm{~m}, 2 \mathrm{H}), 2.32(\mathrm{t}, \mathrm{J}=7.5 \mathrm{~Hz}, 2 \mathrm{H}), 2.03(\mathrm{~s}, 3 \mathrm{H}), 2.02-1.82(\mathrm{~m}$, $6 \mathrm{H}), 1.75-1.22(\mathrm{~m}, 30 \mathrm{H}), 1.19(\mathrm{~d}, \mathrm{~J}=5.6 \mathrm{~Hz}, 3 \mathrm{H}), 0.80(\mathrm{t}, \mathrm{J}=7.1 \mathrm{~Hz}, 3 \mathrm{H}) ;{ }^{13} \mathrm{C}$ NMR $(125$ $\left.\mathrm{MHz}, \mathrm{CDCl}_{3}\right)$ 180.6, 173.5, 171.1, 163.9, 163.0, 150.0, 133.0, 132.3, 129.7, 118.1, 112.5, 86.2, 77.2, 71.6, 70.3, 66.6, 64.9, 59.1, 37.7, 35.6, 35.4, 34.8, 34.2, 33.3, 29.63, 29.61, 29.56, 29.5, 29.4, 29.3, 29.23, 29.19, 29.11, 29.07, 28.6, 26.1, 24.9, 24.2, 21.5, 20.1, 10.0; IR (neat) 2927, 2856, 1729, 1663, 1617, 1563, 1463, 1291, $1256 \mathrm{~cm}^{-1} ;[\alpha]_{589}^{27}-32.4$, $[\alpha]_{577}^{27}-39.4,[\alpha]_{546}^{27}-46.2,[\alpha]^{27}{ }_{435}-108,[\alpha]^{27}{ }_{405}-132\left(c\right.$ 0.72, MeOH); HRMS (ESI $\left.{ }^{+}\right)$ $\mathrm{m} / \mathrm{z} 738.5041$ (738.5057 calculated for $\mathrm{C}_{43} \mathrm{H}_{68} \mathrm{~N}_{3} \mathrm{O}_{7}\left[\mathrm{M}^{+}\right]$).

Preparation of tetracyclic acid 18. A solution of allyl ester 16 (27 mg, 0.035 $\mathrm{mmol})$, tetrakis(triphenylphosphine)palladium $(17 \mathrm{mg}, 0.014 \mathrm{mmol})$, and morpholine (11 $\mu \mathrm{L}, 0.11 \mathrm{mmol})$ was maintained in $\mathrm{MeCN}(0.3 \mathrm{~mL}$, degassed with $\mathrm{Ar})$ at $\mathrm{rt}$ for $2 \mathrm{~h}$ under Ar. The reaction mixture was diluted with $\mathrm{MeCN}(10 \mathrm{~mL})$, filtered through a pad of Celite and concentrated. The residual oil was partitioned between $\mathrm{CHCl}_{3}(30 \mathrm{~mL})$ and $1 \mathrm{~N} \mathrm{HCl}(7 \mathrm{~mL})$. The organic layer was washed with $1 \mathrm{~N} \mathrm{HCl}(5 \mathrm{~mL})$ and brine $(2 \times 5 \mathrm{~mL})$, dried over anhydrous $\mathrm{Na}_{2} \mathrm{SO}_{4}$ and concentrated. The residual oil was purified by reverse phase HPLC (Phenomenex C-18, 10ml/min, $10 \% \mathrm{MeCN}: 0.1 \% \mathrm{HCl}$ in $\mathrm{H}_{2} \mathrm{O}$ to $90 \% \mathrm{MeCN}: 0.1 \% \mathrm{HCl}$ in $\mathrm{H}_{2} \mathrm{O}$ over $\left.20 \mathrm{~min}, \mathrm{tR}=21 \mathrm{~min}\right)$ to give 18 as clear oil $(21 \mathrm{mg}, 0.026 \mathrm{mmol}, 75 \%$ yield $):{ }^{1} \mathrm{H}$ $\operatorname{NMR}\left(500 \mathrm{MHz}, \mathrm{CD}_{3} \mathrm{OD}\right): \delta$ 5.73-5.77 (m, 1H), 5.55 (br d, J = $\left.11 \mathrm{~Hz}, 1 \mathrm{H}\right), 4.88(\mathrm{~m}, 2 \mathrm{H})$, $4.57(\mathrm{~m}, 1 \mathrm{H}), 4.37(\mathrm{t}, \mathrm{J}=6.5 \mathrm{~Hz}, 2 \mathrm{H}), 3.55-3.65(\mathrm{~m}, 1 \mathrm{H}), 3.15-3.25(\mathrm{~m}, 1 \mathrm{H}), 3.14-3.08(\mathrm{~m}$, $1 \mathrm{H}), 2.85(\mathrm{dd}, \mathrm{J}=13.2,3.6 \mathrm{~Hz}, 1 \mathrm{H}), 2.78-2.72(\mathrm{~m}, 1 \mathrm{H}), 2.71-2.66(\mathrm{~m}, 1 \mathrm{H}), 2.66-2.62(\mathrm{~m}$, $1 \mathrm{H}), 2.27(\mathrm{t}, \mathrm{J}=7.4 \mathrm{~Hz}, 2 \mathrm{H}), 2.25-2.18(\mathrm{~m}, 1 \mathrm{H}), 2.15-2.08(\mathrm{~m}, 1 \mathrm{H}), 2.02-2.06(\mathrm{~m}, 1 \mathrm{H}), 2.00$ $(\mathrm{s}, 3 \mathrm{H}), 1.85-1.75(\mathrm{~m}, 4 \mathrm{H}), 1.72-1.45(\mathrm{~m}, 8 \mathrm{H}), 1.25-1.38(\mathrm{~m}, 23 \mathrm{H}), 1.20(\mathrm{~d}, \mathrm{~J}=6.2 \mathrm{~Hz}, 3 \mathrm{H})$, $0.81(\mathrm{t}, \mathrm{J}=7.3 \mathrm{~Hz}, 3 \mathrm{H}) ;{ }^{13} \mathrm{C} \mathrm{NMR}\left(125 \mathrm{MHz}, \mathrm{CD}_{3} \mathrm{OD}\right): \delta 181.6,177.7,172.6,167.6,164.7$, $151.8,133.8,131.6,114.6,87.0,72.7,71.9,67.6,61.0,38.4,37.7,36.6,35.8,35.1,34.6$, $30.89,30.85,30.84,30.79,30.75,30.58,30.50,30.4,30.3,29.8,27.4,26.2,25.3,25.0,21.4$, 20.5, 10.7; IR (neat) 2924, 2853, 1719, 1624, 1577, 1252, 1127, 1118, $1026 \mathrm{~cm}^{-1}$; $[\alpha]^{27}{ }_{589}-$ 32.4, $[\alpha]^{27}{ }_{577}-39.4,[\alpha]_{546}^{27}-46.2,[\alpha]^{27}{ }_{435}-108,[\alpha]^{27}{ }_{405}-132(c$ 0.72, MeOH); HRMS $\left(\mathrm{ESI}^{+}\right) \mathrm{m} / \mathrm{z} 698.4759$ (698.4744 calculated for $\left.\mathrm{C}_{40} \mathrm{H}_{64} \mathrm{~N}_{3} \mathrm{O}_{7}\left[\mathrm{M}^{+}\right]\right)$.

Preparation of compound $N$-((3S)-4-\{[3-(acetylamino)propyl]amino $\}-3-$ hydroxybutyl)acetamide (19) from compound 20. To a solution of $\mathbf{2 0}^{1}(95 \mathrm{mg}, 0.21$ mmol) in $\mathrm{CHCl}_{3}(1 \mathrm{~mL})$ was added trifluoroacetic acid $(0.50 \mathrm{~mL})$ at $0{ }^{\circ} \mathrm{C}$ and the solution was maintained at $0{ }^{\circ} \mathrm{C}$ for $5 \mathrm{~h}$. The solution was concentrated and the residual trifluoroactic acid was removed by azeotropic distillation with heptane. To the resulting crude oil was added pyridine $(1 \mathrm{~mL})$ and acetic anhydride $(0.5 \mathrm{~mL})$ at $0^{\circ} \mathrm{C}$. The resulting solution was stirred at $0^{\circ} \mathrm{C}$ for $16 \mathrm{~h}$, by which time the reaction was complete. Low-boiling compounds were removed under reduced pressure $(\sim 1 \mathrm{~mm})$. To the residual oil was added

\footnotetext{
${ }^{1}$ Coffey, D. S.; McDonald, A. I.; Overman, L. E. J. Org. Chem. 1999, 64, 8741-8742.
} 
$\mathrm{MeOH}(3 \mathrm{~mL})$ and sodium hydroxide $(400 \mathrm{mg}, 10 \mathrm{mmol})$ and this solution was stirred at $\mathrm{rt}$ for $16 \mathrm{~h}$. Low-boiling compounds were removed in vacuo and the residual oil was partitioned between $\mathrm{CH}_{2} \mathrm{Cl}_{2}(30 \mathrm{~mL})$ and water $(30 \mathrm{~mL})$. The aqueous layer was extracted with $\mathrm{CH}_{2} \mathrm{Cl}_{2}$. The organic layers were combined, dried over anh. $\mathrm{MgSO}_{4}$ and concentrated. The residual oil was purified by column chromatography $\left(5 \%\right.$ to $15 \% \mathrm{MeOH}$ in $\left.\mathrm{CHCl}_{3}\right)$ to give the corresponding diacetate as a clear oil $\left(50 \mathrm{mg}, 0.014 \mathrm{mmol}, 71 \%\right.$ yield): ${ }^{1} \mathrm{H}$ NMR $\left(500 \mathrm{MHz}, \mathrm{CD}_{3} \mathrm{OD}\right): \delta$ 7.23-7.30 (m, 5H), $6.64(\mathrm{~m}, 1 \mathrm{H}), 6.45(\mathrm{~m}, 1 \mathrm{H}), 3.75(\mathrm{~d}, \mathrm{~J}=11 \mathrm{~Hz}$, $1 \mathrm{H}), 3.70-3.74(\mathrm{~m}, 1 \mathrm{H}), 3.37(\mathrm{~d}, \mathrm{~J}=11 \mathrm{~Hz}, 1 \mathrm{H}), 3.36(\mathrm{~m}, 1 \mathrm{H}), 3.15-3.22(\mathrm{~m}, 3 \mathrm{H}), 2.54-2.57$ $(\mathrm{m}, 1 \mathrm{H}), 2.34-2.45(\mathrm{~m}, 3 \mathrm{H}), 1.92(\mathrm{~s}, 3 \mathrm{H}), 1.86(\mathrm{~s}, 3 \mathrm{H}), 1.56-1.62(\mathrm{~m}, 2 \mathrm{H}), 1.41-1.50(\mathrm{~m}$, $1 \mathrm{H}) ;{ }^{13} \mathrm{C}$ NMR $\left(125 \mathrm{MHz}, \mathrm{CD}_{3} \mathrm{OD}\right): \delta 170.8,170.3,138.6,129.0,128.3,128.1,66.4,60.0$, 59.1, 51.7, 37.6, 36.8, 34.1, 26.4, 23.1; IR (neat) 3325, 2926, 2855, 1644, 1224, 1145, 1109, $1027 \mathrm{~cm}^{-1} ;[\alpha]^{27}{ }_{589}-10.3,[\alpha]^{27}{ }_{577}-3.4,[\alpha]_{546}^{27}-5.5,[\alpha]^{27}{ }_{435}-9.2,[\alpha]^{27}{ }_{405}-13.3(c 0.92$, $\mathrm{CH}_{2} \mathrm{Cl}_{2}$ ); HRMS (ESI ${ }^{+}$) m/z 336.2295 (336.2287 calculated for $\mathrm{C}_{18} \mathrm{H}_{30} \mathrm{~N}_{3} \mathrm{O}_{3}\left[\mathrm{M}+\mathrm{H}^{+}\right]$).

A mixture of this product (50 mg, $0.149 \mathrm{mmol}), \mathrm{Pd} / \mathrm{C}(10 \%, 18 \mathrm{mg})$ and $\mathrm{MeOH}(5$ $\mathrm{mL})$ was stirred under hydrogen $(1 \mathrm{~atm})$ for $5 \mathrm{~h}$. The mixture was filtered through a pad of Celite, and the plug was washed with $\mathrm{MeOH}(10 \times 3 \mathrm{~mL})$. The combined eluent was concentrated to give 19 (34 mg, $0.14 \mathrm{mmol}$, 95\% yield), which was used without further purification for the next step: ${ }^{1} \mathrm{H}$ NMR $\left(500 \mathrm{MHz}, \mathrm{CD}_{3} \mathrm{OD}\right): \delta 3.78-3.84(\mathrm{~m}, 1 \mathrm{H}), 2.72-2.82$ $(\mathrm{m}, 3 \mathrm{H}), 2.70(\mathrm{~m}, 1 \mathrm{H}), 1.98(\mathrm{~s}, 6 \mathrm{H}), 1.80(\mathrm{t}, \mathrm{J}=6.3 \mathrm{~Hz}, 2 \mathrm{H}), 1.68(\mathrm{~m}, 1 \mathrm{H}), 1.60(\mathrm{~m}, 1 \mathrm{H})$; ${ }^{13} \mathrm{C}$ NMR (125 MHz, CD $\left.3 \mathrm{OD}\right): \delta$ 172.6, 172.5, 66.9, 54.7, 47.7, 47.5, 46.4, 36.9, 36.1, 34.9, 28.4, 21.6; IR (neat) 3332, 2927, 2858, 1654, 1222, 1165, 1112, $1029 \mathrm{~cm}^{-1} ;[\alpha]^{27}{ }_{589}+6.0$, $[\alpha]_{57}^{27}+4.4,[\alpha]_{546}^{27}-1.6,[\alpha]_{435}^{27}-23.2,[\alpha]_{405}^{27}-39.3\left(c 0.92, \mathrm{CH}_{2} \mathrm{Cl}_{2}\right) ; \mathrm{HRMS}\left(\mathrm{ESI}^{+}\right) \mathrm{m} / \mathrm{z}$ 246.1832 (246.1818 calculated for $\left.\mathrm{C}_{11} \mathrm{H}_{24} \mathrm{~N}_{3} \mathrm{O}_{3}\left[\mathrm{M}+\mathrm{H}^{+}\right]\right)$.

Diamide 21. To a solution of acid $18(13 \mathrm{mg}, 0.018 \mathrm{mmol})$ in DMF $(0.5 \mathrm{~mL})$ was added $19(5 \mathrm{mg}, 0.027 \mathrm{mmol})$, triethylamine $(15 \mu \mathrm{L}, 0.090 \mathrm{mmol})$, and the BOP reagent (12 $\mathrm{mg}, 0.027 \mathrm{mmol})$ and the solution was maintained at $\mathrm{rt}$ for $1 \mathrm{~h}$. The reaction was diluted with ether $(20 \mathrm{~mL})$ and washed with saturated $\mathrm{NH}_{4} \mathrm{Cl}$ solution $(5 \mathrm{~mL})$ and brine $(5 \mathrm{~mL})$. The organic layer was dried over anhdrous $\mathrm{Na}_{2} \mathrm{SO}_{4}$ and concentrated. The residual oil was purified by reverse-phase HPLC (Phenomenex C-18, 10ml/min, 10\% MeCN:0.1\% $\mathrm{HCl}$ in $\mathrm{H}_{2} \mathrm{O}$ to $90 \% \mathrm{MeCN}: 0.1 \% \mathrm{HCl}$ in $\mathrm{H}_{2} \mathrm{O}$ over $20 \mathrm{~min}, \mathrm{tR}=20.5 \mathrm{~min}$ ) to give 21 as a colorless foam (8 mg, $0.009 \mathrm{mmol}, 50 \%$ yield): ${ }^{1} \mathrm{H}$ NMR (500 MHz, $\left.\mathrm{CD}_{3} \mathrm{OD}\right): \delta$ 5.73-5.77 $(\mathrm{m}, 1 \mathrm{H})$, $5.55(\mathrm{~m}, 1 \mathrm{H}), 4.88(\mathrm{~m}, 2 \mathrm{H}), 4.57(\mathrm{~m}, 1 \mathrm{H}), 4.37(\mathrm{t}, \mathrm{J}=6.5 \mathrm{~Hz}, 2 \mathrm{H}), 3.98(\mathrm{~m}, 1 \mathrm{H}), 3.44-3.62$ $(\mathrm{m}, 3 \mathrm{H}), 3.34-3.42(\mathrm{~m}, 2 \mathrm{H}), 3.18-3.25(\mathrm{~m}, 5 \mathrm{H}), 3.08-3.17(\mathrm{~m}, 2 \mathrm{H}), 2.85(\mathrm{dd}, \mathrm{J}=13.2,3.6$ $\mathrm{Hz}, 1 \mathrm{H}), 2.70-2.77(\mathrm{~m}, 1 \mathrm{H}), 2.62-2.68(\mathrm{~m}, 1 \mathrm{H}), 2.43-2.52(\mathrm{~m}, 1 \mathrm{H}), 2.30-2.40(\mathrm{~m}, 2 \mathrm{H}), 2.21-$ $2.23(\mathrm{~m}, 1 \mathrm{H}), 2.04-2.17(\mathrm{~m}, 2 \mathrm{H}), 2.03(\mathrm{~s}, 3 \mathrm{H}), 1.95(\mathrm{~s}, 3 \mathrm{H}), 1.93(\mathrm{~s}, 3 \mathrm{H}), 1.65-1.85(\mathrm{~m}, 9 \mathrm{H})$, $1.42-1.62(\mathrm{~m}, 8 \mathrm{H}), 1.25-1.38(\mathrm{~m}, 20 \mathrm{H}), 1.20(\mathrm{~d}, \mathrm{~J}=6.2 \mathrm{~Hz}, 3 \mathrm{H}), 0.82(\mathrm{t}, \mathrm{J}=7.3 \mathrm{~Hz}, 3 \mathrm{H})$; ${ }^{13} \mathrm{C}$ NMR (125 MHz, $\left.\mathrm{CD}_{3} \mathrm{OD}\right): \delta 181.6,177.7,177.5,172.6,167.6,164.7,151.8,133.8$, 131.6, 114.6, 87.0, 72.7, 71.9, 68.5, 67.6, 61.0, 54.9, 43.9 38.4, 38.3, 37.7, 36.6, 35.8, 35.1, $34.6,32.9,30.89,30.85,30.84,30.79,30.75,30.58,30.50,30.4,30.3,29.8,27.4,26.6,26.2$, 25.3, 25.0, 21.4, 20.5, 10.7; IR (neat) 3254, 2927, 2856, 1730, 1636, 1570, 1450, 1375, $1268,1259,1128,1116 \mathrm{~cm}^{-1} ;[\alpha]^{27}{ }_{589}-34.2,[\alpha]^{27}{ }_{577}-39.6,[\alpha]^{27}{ }_{546}-43.7,[\alpha]^{27}{ }_{435}-66.3$, 
$[\alpha]^{27}{ }_{405}-97.1\left(c \quad 0.45, \mathrm{CH}_{2} \mathrm{Cl}_{2}\right.$ ); HRMS $\left(\mathrm{ESI}^{+}\right) \mathrm{m} / \mathrm{z} 925.6177$ (925.6378 calculated for $\left.\mathrm{C}_{51} \mathrm{H}_{85} \mathrm{~N}_{6} \mathrm{O}_{9}\left[\mathrm{M}^{+}\right]\right)$.

Diamide 22. To a solution of acid $18(19 \mathrm{mg}, 0.026 \mathrm{mmol})$ in $\mathrm{CH}_{2} \mathrm{Cl}_{2}(0.5 \mathrm{~mL})$ was added hydroxyspermidine derivative $\mathbf{2 0}^{1}(14 \mathrm{mg}, 0.039 \mathrm{mmol})$, triethylamine $(18 \mu \mathrm{L}$, $0.13 \mathrm{mmol}$ ), and the BOP reagent $(22 \mathrm{mg}, 0.039 \mathrm{mmol}$ ), and the resulting solution was maintained at $\mathrm{rt}$ for $1 \mathrm{~h}$. The solution was diluted with ether $(20 \mathrm{~mL})$ and washed with saturated $\mathrm{NH}_{4} \mathrm{Cl}$ solution $(5 \mathrm{~mL})$ and brine $(5 \mathrm{~mL})$. The organic layer was dried over anhdrous $\mathrm{Na}_{2} \mathrm{SO}_{4}$ and concentrated. The residual oil was purified by reverse-phase HPLC (Phenomenex C-18, $10 \mathrm{ml} / \mathrm{min}, 10 \% \mathrm{MeCN}: 0.1 \% \mathrm{HCl}$ in $\mathrm{H}_{2} \mathrm{O}$ to $90 \% \mathrm{MeCN}: 0.1 \% \mathrm{HCl}$ in $\mathrm{H}_{2} \mathrm{O}$ over $\left.20 \mathrm{~min}, \mathrm{tR}=24.6 \mathrm{~min}\right)$ to give 22 as a colorless foam $(22 \mathrm{mg}, 0.021 \mathrm{mmol}, 80 \%$ yield): ${ }^{1} \mathrm{H}$ NMR $\left(500 \mathrm{MHz}, \mathrm{CD}_{3} \mathrm{OD}\right): \delta$ 5.97-5.89 (m, 1H), $5.76(\mathrm{~m}, 1 \mathrm{H}), 5.55$ (br d, J = $11.2 \mathrm{~Hz}, 1 \mathrm{H}), 5.30(\mathrm{br} \mathrm{d}, \mathrm{J}=17.1 \mathrm{~Hz}, 1 \mathrm{H}), 5.20(\mathrm{br} \mathrm{d}, \mathrm{J}=10.5 \mathrm{~Hz}, 1 \mathrm{H}), 4.86(\mathrm{~m}, 1 \mathrm{H}), 4.56$ $(\mathrm{m}, 3 \mathrm{H}), 4.37(\mathrm{t}, \mathrm{J}=6.5 \mathrm{~Hz}, 2 \mathrm{H}), 3.75(\mathrm{~m}, 1 \mathrm{H}), 3.68-3.52(\mathrm{~m}, 2 \mathrm{H}), 3.21-3.19(\mathrm{~m}, 1 \mathrm{H}), 3.14-$ $3.12(\mathrm{~m}, 1 \mathrm{H}), 2.85(\mathrm{dd}, \mathrm{J}=13.4,3.6 \mathrm{~Hz}, 1 \mathrm{H}), 2.78-2.72(\mathrm{~m}, 1 \mathrm{H}), 2.66-2.62(\mathrm{~m}, 1 \mathrm{H}), 2.55-$ $2.45(\mathrm{~m}, 1 \mathrm{H}), 2.34(\mathrm{t}, \mathrm{J}=7.2 \mathrm{~Hz}, 2 \mathrm{H}), 2.25-2.18(\mathrm{~m}, 1 \mathrm{H}), 2.15-2.02(\mathrm{~m}, 2 \mathrm{H}), 1.95-1.75(\mathrm{~m}$, 4H), $1.70(\operatorname{app~t}, \mathrm{J}=13.0 \mathrm{~Hz}, 1 \mathrm{H}), 1.61-1.31(\mathrm{~m}, 28 \mathrm{H}), 1.16(\mathrm{~d}, \mathrm{~J}=6.2 \mathrm{~Hz}, 3 \mathrm{H}), 0.83$ (t, J $=6.3 \mathrm{~Hz}, 3 \mathrm{H}) ;{ }^{13} \mathrm{C} \mathrm{NMR}\left(125 \mathrm{MHz}, \mathrm{CD}_{3} \mathrm{OD}\right): \delta 181.6,176.7,176.3,172.6,167.6,164.7$, $151.8,133.8,131.6,114.6,87.0,80.1,72.7,71.9,69.2,68.5,67.6,61.0,55.1,53.6,38.4$, $37.7,36.6,35.7,34.7,30.89,30.87,30.84,30.82,30.80,30.71,30.5,30.4,30.3,29.8,29.0$, 27.4, 25.3, 25.0, 21.4, 20.5, 10.7; IR (neat) 3329, 2927, 2854, 1707, 1629, 1459, 1364, $1273,1254,1170,1116 \mathrm{~cm}^{-1} ;[\alpha]_{589}^{27}-27.6,[\alpha]^{27}{ }_{577}-29.1,[\alpha]^{27}{ }_{546}-32.0,[\alpha]^{27}{ }_{435}-51.7$, $[\alpha]_{405}^{27}-59.1$ (c $\left.0.82, \mathrm{MeOH}\right) ; \mathrm{HRMS}\left(\mathrm{ESI}^{+}\right) \mathrm{m} / \mathrm{z} 1042.9$ (1042.7 calculated for $\left.\mathrm{C}_{57} \mathrm{H}_{97} \mathrm{~N}_{6} \mathrm{O}_{11}\left[\mathrm{M}^{+}\right]\right)$.

(43S)-peracetylcrambidine (5a). To a stirred solution of amide 22 (14 $\mathrm{mg}, 0.013$ $\mathrm{mmol})$ in EtOAc solution $(0.2 \mathrm{~mL})$ was added $3 \mathrm{M} \mathrm{HCl}(20 \mu \mathrm{L})$ at $0^{\circ} \mathrm{C}$ and the resulting solution was stirred at $0^{\circ} \mathrm{C}$ for $2 \mathrm{~h}$. EtOAc was removed by concentration and the residue was washed with EtOAc $(1 \mathrm{~mL})$. The residual oil was dried under reduced pressure and used for the next step without further purification. To this crude oil was added pyridine $(0.1$ $\mathrm{mL})$ and acetic anhydride $(0.1 \mathrm{~mL})$ at $0^{\circ} \mathrm{C}$, and the resulting solution was maintained at $\mathrm{rt}$ for $24 \mathrm{~h}$. The reaction was purified directly by reverse-phase HPLC (Phenomenex C-18, $10 \mathrm{ml} / \mathrm{min}, 10 \% \mathrm{MeCN}: 0.1 \% \mathrm{HCl}$ in $\mathrm{H}_{2} \mathrm{O}$ to $90 \% \mathrm{MeCN}: 0.1 \% \mathrm{HCl}$ in $\mathrm{H}_{2} \mathrm{O}$ over $20 \mathrm{~min}$, tR $=21.2 \mathrm{~min}$ ) to give $\mathbf{5 a}$ as clear oil $(5.6 \mathrm{mg}, 0.052 \mathrm{mmol}, 40 \%$ yield $):$ The ${ }^{1} \mathrm{H}$ and ${ }^{13} \mathrm{C}$ NMR spectra taken in $\mathrm{CDCl}_{3}$ matched those of reported data for natural peracetylcrambidine: IR (neat) $3254,2927,2856,1730,1636,1570,1450,1375,1268,1259,1128,1116 \mathrm{~cm}^{-1}$; uv $(\mathrm{MeOH}) \lambda \max 254(19,000), 299(4,900) ;[\alpha]^{27}{ }_{589}-37.2,[\alpha]^{27}{ }_{577}-38.6,[\alpha]^{27}{ }_{546}-41.7$, $\left.[\alpha]_{435}^{27}-65.3,[\alpha]_{405}^{27}-109(c) 1.4, \mathrm{CH}_{2} \mathrm{Cl}_{2}\right)$; HRMS $\left(\mathrm{ESI}^{+}\right) \mathrm{m} / \mathrm{z} 967.6496(967.6484$ calculated for $\mathrm{C}_{53} \mathrm{H}_{87} \mathrm{~N}_{6} \mathrm{O}_{10}\left[\mathrm{M}^{+}\right]$). 
(43R)-peracetylcrambidine (5b). Using an analogous procedure, acid 18 (16 $\mathrm{mg}$, $0.022 \mathrm{mmol})$ was allowed to react with hydroxyspermidine derivative ent-20 ${ }^{2}(12 \mathrm{mg}$, $0.032 \mathrm{mmol})$, the BOP reagent $(14 \mathrm{mg}, 0.032 \mathrm{mmol})$, and triethylamine $(15 \mu \mathrm{L}, 0.11 \mathrm{mmol})$ at $\mathrm{rt}$ for $2 \mathrm{~h}$ to give the $43(R)$-epimer of amide 22 as a colorless oil $(17 \mathrm{mg}, 0.017 \mathrm{mmol}$, $77 \%$ yield). The ${ }^{1} \mathrm{H}$ and ${ }^{13} \mathrm{C}$ NMR spectra of this compound were indistinguishable from those of 22. IR (neat) $3329,2927,2854,1711,1629,1273,1254,1169,1116 \mathrm{~cm}^{-1} ;[\alpha]^{27}{ }_{589}$ $-34.1,[\alpha]^{27}{ }_{577}-35.5,[\alpha]^{27}{ }_{546}-38.1,[\alpha]^{27}{ }_{435}-61.4,[\alpha]^{27}{ }_{405}-81.5(c 0.56, \mathrm{MeOH})$. HRMS $\left(\mathrm{ESI}^{+}\right) \mathrm{m} / \mathrm{z} 1042.8$ (1042.7 calculated for $\mathrm{C}_{57} \mathrm{H}_{97} \mathrm{~N}_{6} \mathrm{O}_{11}\left[\mathrm{M}^{+}\right]$).

This amide $(15 \mathrm{mg}, 0.0145 \mathrm{mmol})$ was deprotected and acetylated to give $\mathbf{5 b}(6.2$ $\mathrm{mg}, 0.0058 \mathrm{mmol}, 40 \%$ yield). The ${ }^{1} \mathrm{H}$ and ${ }^{13} \mathrm{C} \mathrm{NMR}$ spectra taken in $\mathrm{CDCl}_{3}$ matched those of reported for natural peracetylcrambidine as well as those for synthetic 5a. IR (neat) 3329, $2927,2854,1725,1581,1555,1473,1374,1257,1129,1118 \mathrm{~cm}^{-1} ;[\alpha]^{27}{ }_{589}-47.5,[\alpha]^{27}{ }_{577}-$ 49.7, $[\alpha]^{27}{ }_{546}-55.3,[\alpha]^{27}{ }_{435}-87.1,[\alpha]^{27}{ }_{405}-98.2(c 0.35, \mathrm{MeOH}) ; \mathrm{HRMS}\left(\mathrm{ESI}^{+}\right) \mathrm{m} / \mathrm{z}$ 967.6496 (967.6484 calculated for $\mathrm{C}_{53} \mathrm{H}_{87} \mathrm{~N}_{6} \mathrm{O}_{10}\left[\mathrm{M}^{+}\right]$).

C19-epimer 23. A solution of alcohol 15 (51 $\mathrm{mg}, 0.070 \mathrm{mmol})$, pyridinium chlorochromate $(23 \mathrm{mg}, 0.10 \mathrm{mmol}), 4 \mathrm{~A}$ molecular sieve $(22 \mathrm{mg})$, and sodium acetate (8 $\mathrm{mg}, 0.10 \mathrm{mmol}$ ) was maintained at $\mathrm{rt}$ for $2 \mathrm{~h}$. The solution then was filtered through a pad of Celite and concentrated. The crude oil was immediately diluted with $\mathrm{MeOH}(1 \mathrm{~mL})$ and cooled to $0^{\circ} \mathrm{C}$. Sodium borohydride $(7.6 \mathrm{mg}, 0.20 \mathrm{mmol})$ was added and the reaction mixture was stirred for $1 \mathrm{~h}$ at $0^{\circ} \mathrm{C}$. The reaction was diluted with $\mathrm{CHCl}_{3}(5 \mathrm{~mL}), 0.1 \mathrm{M} \mathrm{HCl}$ $(1 \mathrm{~mL})$ and the mixture was vigorously stirred for at $\mathrm{rt} 1 \mathrm{~h}$. The organic phase was separated and the aqueous phase was extracted with $\mathrm{CHCl}_{3}(3 \times 10 \mathrm{~mL})$. The organic layers were combined, dried over anh. $\mathrm{MgSO}_{4}$ and concentrated. The residual oil was purified by flash chromatography $\left(\mathrm{CHCl}_{3}\right.$ to $1 \% \mathrm{MeOH}$ in $\left.\mathrm{CHCl}_{3}\right)$ to give the starting alcohol $15(9 \mathrm{mg}$, $0.010 \mathrm{mmol})$. Further elution $\left(2 \% \mathrm{MeOH}\right.$ in $\left.\mathrm{CHCl}_{3}\right)$ provided epimer 23 as a clear oil (10 $\mathrm{mg}, 0.014 \mathrm{mmol}, 20 \%$ yield). In addition to the pure compounds, a mixture of two diastereomers was also obtained $(\sim 17 \mathrm{mg})$. C-19 Epimer 23: ${ }^{1} \mathrm{H}$ NMR (500 MHz, $\left.\mathrm{CD}_{3} \mathrm{OD}\right)$ : d 5.97-5.89 (m, 1H), $5.76(\mathrm{~m}, 1 \mathrm{H}), 5.55$ (br d, J = 11.2 Hz, 1H), 5.30 (br d, J = 17.1 Hz, 1H), 5.20 (br d, J = $10.5 \mathrm{~Hz}, 1 \mathrm{H}), 4.86(\mathrm{~m}, 1 \mathrm{H}), 4.56(\mathrm{~m}, 3 \mathrm{H}), 4.37(\mathrm{t}, \mathrm{J}=6.5 \mathrm{~Hz}, 2 \mathrm{H}), 3.75(\mathrm{~m}$, $1 \mathrm{H}), 3.68-3.52(\mathrm{~m}, 2 \mathrm{H}), 3.18(\mathrm{~m}, 2 \mathrm{H}), 2.85(\mathrm{dd}, \mathrm{J}=13.4,3.6 \mathrm{~Hz}, 1 \mathrm{H}), 2.78-2.72(\mathrm{~m}, 1 \mathrm{H})$, 2.66-2.62 (m, 1H), 2.55-2.45 (m, 1H), $2.34(\mathrm{t}, \mathrm{J}=7.2 \mathrm{~Hz}, 2 \mathrm{H}), 2.25-2.18(\mathrm{~m}, 1 \mathrm{H}), 2.15-$ $2.02(\mathrm{~m}, 2 \mathrm{H}), 1.95-1.75(\mathrm{~m}, 4 \mathrm{H}), 1.70(\operatorname{app~t}, \mathrm{J}=13.0 \mathrm{~Hz}, 1 \mathrm{H}), 1.61-1.31(\mathrm{~m}, 28 \mathrm{H}), 1.16(\mathrm{~d}$, $\mathrm{J}=6.2 \mathrm{~Hz}, 3 \mathrm{H}), 0.83(\mathrm{t}, \mathrm{J}=6.3 \mathrm{~Hz}, 3 \mathrm{H}) ;{ }^{13} \mathrm{C} \mathrm{NMR}\left(125 \mathrm{MHz}, \mathrm{CD}_{3} \mathrm{OD}\right): \delta$ 181.9, 175.2, $167.5,164.7,151.8,133.9,133.8,131.6,118.3,114.6,87.0,72.7,68.3,67.5,66.1,61.0$, $39.7,38.7,37.7,35.1,30.89,30.87,30.84,30.82,30.80,30.71,30.5,30.4,30.3,29.8,27.4$, 26.2, 25.7, 23.8, 10.7; IR (neat) 3543, 2925, 2856, 1722, 1626, 1579, 1451, 1258, 1119, $988 \mathrm{~cm}^{-1} ;[\alpha]_{589}^{27}-18.7,[\alpha]_{577}^{27}-19.4,[\alpha]_{546}^{27}-20.6,[\alpha]_{435}^{27}-31.6,[\alpha]^{27}{ }_{405}-57.5(c 0.18$, $\mathrm{MeOH}) ; \mathrm{HRMS}\left(\mathrm{ESI}^{+}\right) \mathrm{m} / \mathrm{z} 696.4961$ (696.4952 calculated for $\mathrm{C}_{41} \mathrm{H}_{66} \mathrm{~N}_{3} \mathrm{O}_{6}\left[\mathrm{M}^{+}\right]$).

\footnotetext{
2 Coffey, D. S.; McDonald, A. I.; Overman, L. E.; Rabinowitz, M. H.; Renhowe, P. A. J. Am. Chem. Soc. 2000, 122, 4893-4903.
} 
C19-epimer 24. A mixture of alcohol 23 (10 mg, $0.014 \mathrm{mmol})$, pyridine $(0.3 \mathrm{~mL})$ and acetic anhydride $(0.2 \mathrm{~mL})$ was maintainted at $\mathrm{rt}$ for $5 \mathrm{~h}$. The mixture purified directly by reverse phase HPLC (Phenomenex C- $18,10 \mathrm{ml} / \mathrm{min}, 10 \% \mathrm{MeCN}: 0.1 \% \mathrm{HCl}$ in $\mathrm{H}_{2} \mathrm{O}$ to $90 \% \mathrm{MeCN}: 0.1 \% \mathrm{HCl}$ in $\mathrm{H}_{2} \mathrm{O}$ over $20 \mathrm{~min}, \mathrm{tR}=21.2 \mathrm{~min}$ ) to give 24 as a clear oil $(8.3 \mathrm{mg}$, $0.0106 \mathrm{mmol}, 80 \%$ yield): ${ }^{1} \mathrm{H}$ NMR $\left(500 \mathrm{MHz}, \mathrm{CDCl}_{3}\right) \delta 12.3(\mathrm{br} \mathrm{s}, 1 \mathrm{H}), 5.87-5.95(\mathrm{~m}$, $1 \mathrm{H}), 5.68(\mathrm{~m}, 1 \mathrm{H}), 5.52(\operatorname{app~d}, \mathrm{J}=10.9 \mathrm{~Hz}, 1 \mathrm{H}), 5.32(\mathrm{dq}, \mathrm{J}=17.2,1.5 \mathrm{~Hz}, 1 \mathrm{H}), 5.27(\mathrm{dq}$, $\mathrm{J}=10.4,1.4 \mathrm{~Hz}, 1 \mathrm{H}), 5.21(\mathrm{br} \mathrm{s}, 1 \mathrm{H}), 4.88(\mathrm{~m}, 1 \mathrm{H}), 4.80(\mathrm{~m}, 1 \mathrm{H}), 4.57(\operatorname{app~d}, \mathrm{J}=5.7 \mathrm{~Hz}$, 2H), 4.32 (br s, 2H), $3.65(\mathrm{~m}, 2 \mathrm{H}), 3.38(\mathrm{~m}, 1 \mathrm{H}), 3.10-3.15(\mathrm{~m}, 2 \mathrm{H}), 2.78(\mathrm{~m}, 1 \mathrm{H}), 2.69(\mathrm{~m}$, $1 \mathrm{H}), 2.34-2.28(\mathrm{~m}, 2 \mathrm{H}), 2.32(\mathrm{t}, \mathrm{J}=7.5 \mathrm{~Hz}, 2 \mathrm{H}), 2.03(\mathrm{~s}, 3 \mathrm{H}), 2.02-1.82(\mathrm{~m}, 6 \mathrm{H}), 1.75-1.22$ $(\mathrm{m}, 30 \mathrm{H}), 1.19(\mathrm{~d}, \mathrm{~J}=5.6 \mathrm{~Hz}, 3 \mathrm{H}), 0.79(\mathrm{t}, \mathrm{J}=7.1 \mathrm{~Hz}, 3 \mathrm{H}) ;{ }^{13} \mathrm{C}$ NMR $\left(125 \mathrm{MHz}, \mathrm{CDCl}_{3}\right)$ 180.6, 173.5, 171.1, 163.9, 163.0, 150.0, 133.0, 132.3, 129.7, 118.1, 112.5, 86.2, 77.2, 71.6, $70.3,66.6,64.9,59.1,37.7,35.6,35.4,34.8,34.2,33.3,29.63,29.61,29.56,29.5,29.4$, $29.3,29.23,29.19,29.11,29.07,28.6,26.1,24.9,24.2,24.0,21.5,20.1,10.0$; IR (neat) $2925,2854,1727,1628,1582,1449,1374,1291,1253 \mathrm{~cm}^{-1} ;[\alpha]^{27}{ }_{589}-35.5,[\alpha]^{27}{ }_{577}-38.3$, $[\alpha]_{546}^{27}-37.3,[\alpha]^{27} 435-56.3,[\alpha]^{27} 405-79.5(c \quad 0.72, \mathrm{MeOH}) ; \mathrm{HRMS}\left(\mathrm{ESI}^{+}\right) \mathrm{m} / \mathrm{z} 738.5038$ (738.5057 calculated for $\mathrm{C}_{43} \mathrm{H}_{68} \mathrm{~N}_{3} \mathrm{O}_{7}\left[\mathrm{M}^{+}\right]$).

C19 epimer of acid 18. By using a procedure analogous to that employed to prepare acid 18, reaction of allyl ester $\mathbf{2 4}(14 \quad \mathrm{mg}, \quad 0.019 \quad \mathrm{mmol})$, tetrakis(triphenylphosphine)palladium $(7.6 \mathrm{mg}, 0.0072 \mathrm{mmol})$, and morpholine $(4.7 \mu \mathrm{L}$, $0.054 \mathrm{mmol})$ gave the title compound as a clear oil $(10 \mathrm{mg}, 0.013 \mathrm{mmol}, 75 \%$ yield). The ${ }^{1} \mathrm{H}$ and ${ }^{13} \mathrm{C}$ spectra of this compound taken in $\mathrm{CD}_{3} \mathrm{OD}$ were indistinguishable from those of the acid 18: $[\alpha]_{589}^{27}-25.4,[\alpha]_{577}^{27}-34.6,[\alpha]_{546}^{27}-40.2,[\alpha]^{27}{ }_{435}-99.2,[\alpha]_{405}^{27}-118(c$ 0.23, $\mathrm{MeOH}) ; \mathrm{HRMS}\left(\mathrm{ESI}^{+}\right) \mathrm{m} / \mathrm{z} 698.4748\left(698.4744\right.$ calculated for $\mathrm{C}_{40} \mathrm{H}_{64} \mathrm{~N}_{3} \mathrm{O}_{7}\left[\mathrm{M}^{+}\right]$).

(43S)-19-epi-peracetylcrambidine (25a). Using a procedure analogous to that employed to prepare 22, a solution of this acid $(11 \mathrm{mg}, 0.015 \mathrm{mmol})$, hydroxyspermidine $\mathbf{2 0}^{1}(8.1 \mathrm{mg}, 0.023 \mathrm{mmol})$, triethylamine $(10 \mu \mathrm{L}, 0.075 \mathrm{mmol})$, and the BOP reagent $(10 \mathrm{mg}$, $0.023 \mathrm{mmol})$ in $\mathrm{CH}_{2} \mathrm{Cl}_{2}(0.5 \mathrm{~mL})$ were allowed to react at $\mathrm{rt}$ for $1 \mathrm{~h}$ to give the corresponding amide as a colorless foam $\left(12 \mathrm{mg}, 0.012 \mathrm{mmol}, 80 \%\right.$ yield): ${ }^{1} \mathrm{H}$ NMR (500 $\left.\mathrm{MHz}, \mathrm{CD}_{3} \mathrm{OD}\right): \delta 5.72-5.78(\mathrm{~m}, 1 \mathrm{H}), 5.55(\mathrm{br} \mathrm{d}, \mathrm{J}=11.1 \mathrm{~Hz}, 1 \mathrm{H}), 4.86-4.93(\mathrm{~m}, 2 \mathrm{H}), 4.55-$ $4.59(\mathrm{~m}, 1 \mathrm{H}), 4.37(\mathrm{t}, \mathrm{J}=6.5 \mathrm{~Hz}, 2 \mathrm{H}), 3.79-3.87(\mathrm{~m}, 1 \mathrm{H}), 3.68-3.61(\mathrm{~m}, 1 \mathrm{H}), 3.52-3.58(\mathrm{~m}$, $1 \mathrm{H}), 3.37-3.49(\mathrm{~m}, 2 \mathrm{H}), 3.27-3.21(\mathrm{~m}, 1 \mathrm{H}), 3.14-3.22(\mathrm{~m}, 4 \mathrm{H}), 3.05-3.07(\mathrm{~m}, 2 \mathrm{H}), 3.02-$ $3.04(\mathrm{~m}, 2 \mathrm{H}), 2.85(\mathrm{dd}, \mathrm{J}=13.4,3.6 \mathrm{~Hz}, 1 \mathrm{H}), 2.78-2.72(\mathrm{~m}, 1 \mathrm{H}), 2.66-2.62(\mathrm{~m}, 1 \mathrm{H}), 2.55-$ $2.45(\mathrm{~m}, 2 \mathrm{H}), 2.36-2.40(\mathrm{~m}, 2 \mathrm{H}), 2.25-2.18(\mathrm{~m}, 1 \mathrm{H}), 2.15-2.02(\mathrm{~m}, 2 \mathrm{H}), 2.00(\mathrm{~s}, 3 \mathrm{H}), 1.95-$ $1.75(\mathrm{~m}, 4 \mathrm{H}), 1.70($ app t, J $=13.0 \mathrm{~Hz}, 1 \mathrm{H}), 1.61-1.27(\mathrm{~m}, 28 \mathrm{H}), 1.30(\mathrm{~s}, 18 \mathrm{H}), 1.22(\mathrm{~d}, \mathrm{~J}=$ $6.2 \mathrm{~Hz}, 3 \mathrm{H}), 0.83(\mathrm{t}, \mathrm{J}=6.3 \mathrm{~Hz}, 3 \mathrm{H}) ;{ }^{13} \mathrm{C}$ NMR $\left(125 \mathrm{MHz}, \mathrm{CD}_{3} \mathrm{OD}\right): \delta 181.6,176.7,176.3$, 172.6, 167.6, 164.7, 151.8, 133.8, 131.6, 114.6, 87.0, 80.1, 72.7, 71.9, 69.2, 68.5, 67.6, 61.0, 55.1, 53.6, 38.4, 37.7, 36.6, 35.7, 34.7, 30.89, 30.87, 30.84, 30.82, 30.80, 30.71, 30.5, 30.4, $30.3,29.8,29.0,27.4,25.3,25.0,21.4,20.5,10.7$; IR (neat) 3317, 2965, 2925, 2853, 1701, $1629,1584,1459,1364,1253,1171 \mathrm{~cm}^{-1} ;[\alpha]_{589}^{27}-23.0,[\alpha]_{577}^{27}-23.3,[\alpha]_{546}^{27}-25.3$, $[\alpha]_{435}^{27}-47.1,[\alpha]_{405}^{27}-65.4\left(c\right.$ 1.7, MeOH); HRMS $\left(\mathrm{ESI}^{+}\right) \mathrm{m} / \mathrm{z} 1042.9$ (1042.7 calculated for $\left.\mathrm{C}_{57} \mathrm{H}_{97} \mathrm{~N}_{6} \mathrm{O}_{11}\left[\mathrm{M}^{+}\right]\right)$. 
Using a procedure analogous to that used to prepare 5a, this amide $(8.6 \mathrm{mg}, 0.0083$ mmol) gave 25a as a clear oil $\left(3.7 \mathrm{mg}, 0.0037 \mathrm{mmol}, 45 \%\right.$ yield): ${ }^{1} \mathrm{H}$ NMR $(500 \mathrm{MHz}$, $\left.\mathrm{CDCl}_{3}\right): \delta 11.2-12.2(\mathrm{~m}, 1 \mathrm{H}), 5.68(\mathrm{~m}, 1 \mathrm{H}), 5.49(\mathrm{br} \mathrm{d}, \mathrm{J}=11 \mathrm{~Hz}, 1 \mathrm{H}), 5.13(\mathrm{~m}, 1 \mathrm{H}), 4.96$ (m, 1H), $4.88(\mathrm{~m}, 1 \mathrm{H}), 4.80(\mathrm{~m}, 1 \mathrm{H}), 4.30(\mathrm{~m}, 2 \mathrm{H}), 3.60-3.65(\mathrm{~m}, 1 \mathrm{H}), 3.50-3.60(\mathrm{~m}, 2 \mathrm{H})$, 3.30-3.50 (m, 4H), 3.15-3.25 (m, 2H), 3.04-3.14 (m, 3H), $2.32(\mathrm{~m}, 3 \mathrm{H}), 2.23(\mathrm{~m}, 2 \mathrm{H}), 1.99$ (s, 6H), $1.97(\mathrm{~s}, 6 \mathrm{H}), 1.98-1.75(\mathrm{~m}, 7 \mathrm{H}), 1.47-1.55(\mathrm{~m}, 4 \mathrm{H}), 1.20-1.40(\mathrm{~m}, 26 \mathrm{H}), 1.17(\mathrm{~d}, \mathrm{~J}$ $=6.6 \mathrm{~Hz}, 3 \mathrm{H}), 0.76(\mathrm{t}, \mathrm{J}=7.3 \mathrm{~Hz}, 3 \mathrm{H}) ;{ }^{13} \mathrm{C} \mathrm{NMR}\left(125 \mathrm{MHz}, \mathrm{CDCl}_{3}\right): \delta 180.6,174.1,173.7$, 171.2, 171.1, 171.0, 164.4, 162.9, 150.1, 132.8, 129.9, 113.0. 86.1, 71.5, 69.3, 66.8, 59.3, $50.5,42.8,38.0,35.8,35.6,33.0,32.0,29.4-29.0,28.6,26.8,26.0,24.1,21.5,20.1,10.1$; $[\alpha]^{27}{ }_{589}-34.7,[\alpha]_{577}^{27}-42.8,[\alpha]_{546}^{27}-55.9,[\alpha]_{435}^{27}-99.4,[\alpha]^{27}{ }_{405}-142(c 0.35, \mathrm{MeOH})$; HRMS $\left(\mathrm{ESI}^{+}\right) \mathrm{m} / \mathrm{z} 967.6478$ (967.6484 calculated for $\left.\mathrm{C}_{53} \mathrm{H}_{87} \mathrm{~N}_{6} \mathrm{O}_{10}\left[\mathrm{M}^{+}\right]\right)$.

(43R)-19-epi-peracetylcrambidine (25b). Using an analogous procedure, the C-19 epimer of acid $18(8.1 \mathrm{mg}, 0.12 \mathrm{mmol})$ was condensed with hydroxyspermidine ent-20 ${ }^{2}$ $(6.0 \mathrm{mg}, 0.017 \mathrm{mmol})$ to give the corresponding amide $(9.5 \mathrm{mg}, 0.0092 \mathrm{mmol}, 80 \%$ yield). NMR data are identical to those of the $43 S$ epimer. IR (neat) 3317, 2965, 2925, 2853, 1701, $1629,1584,1459,1364,1253,1171 \mathrm{~cm}^{-1} ;[\alpha]^{27}{ }_{589}-39.6,[\alpha]^{27}{ }_{577}-45.9[\alpha]^{27}{ }_{546}-80.4$, $[\alpha]^{27}{ }_{435}-122,[\alpha]_{405}^{27}-198\left(c\right.$ 0.46, MeOH); HRMS (ESI $\left.{ }^{+}\right) \mathrm{m} / \mathrm{z} 1042.7$ (1042.7 calculated for $\left.\mathrm{C}_{57} \mathrm{H}_{97} \mathrm{~N}_{6} \mathrm{O}_{11}\left[\mathrm{M}^{+}\right]\right)$.

This amide (10 mg, $0.010 \mathrm{mmol}$ ) was deprotected and acetylated to give $\mathbf{2 5 \mathbf { b }}$ (4.2 $\mathrm{mg}, 0.0042 \mathrm{mmol}, 42 \%$ yield). The ${ }^{1} \mathrm{H}$ and ${ }^{13} \mathrm{C}$ NMR spectra taken in $\mathrm{CDCl}_{3}$ matched those of (43S)-peracetylcrambidine. $[\alpha]^{27}{ }_{589}-34.7,[\alpha]_{577}^{27}-42.8,[\alpha]_{546}^{27}-55.9,[\alpha]^{27} 435-$ 99.4, $[\alpha]^{27}{ }_{405}-142\left(c\right.$ 0.35, MeOH); HRMS $\left(\mathrm{ESI}^{+}\right) \mathrm{m} / \mathrm{z} 967.6488$ (967.6484 calculated for $\left.\mathrm{C}_{53} \mathrm{H}_{87} \mathrm{~N}_{6} \mathrm{O}_{10}\left[\mathrm{M}^{+}\right]\right)$.

Amide 26. To a solution of acid $6(19 \mathrm{mg}, 0.028 \mathrm{mmol})$ in $\mathrm{CH}_{2} \mathrm{Cl}_{2}(0.5 \mathrm{~mL})$ was added hydroxyspermidine $\mathbf{2 0}^{1}(18 \mathrm{mg}, 0.050 \mathrm{mmol})$, triethylamine $(15 \mu \mathrm{L}, \mathrm{mmol})$, and the BOP reagent $(22 \mathrm{mg}, 0.050 \mathrm{mmol})$. The solution was maintained at $\mathrm{rt}$ for $1 \mathrm{~h}$. The resulting solution was diluted with ether $(20 \mathrm{~mL})$ and washed with saturated $\mathrm{NH}_{4} \mathrm{Cl}$ solution $(5 \mathrm{~mL})$ and brine $(5 \mathrm{~mL})$. The organic layer was dried over anhydrous $\mathrm{Na}_{2} \mathrm{SO}_{4}$ and concentrated. The residual oil was purified by reverse-phase HPLC (Phenomex C-18, 10 $\mathrm{ml} / \mathrm{min}, 10 \%$ to $90 \% \mathrm{CH}_{3} \mathrm{CN}: 0.1 \%$ aq $\mathrm{HCl}$ solution, $\mathrm{tR}=23.5 \mathrm{~min}$ ) to give $\mathbf{2 6}$ as a colorless foam $(22 \mathrm{mg}$, $0.021 \mathrm{mmol}, 80 \%$ yield): ${ }^{1} \mathrm{H}$ NMR $\left(500 \mathrm{MHz}, \mathrm{CD}_{3} \mathrm{OD}\right): \delta 5.76($ app t, J $=9.1 \mathrm{~Hz}, 1 \mathrm{H}), 5.55$ $(\mathrm{m}, 1 \mathrm{H}), 4.83(\mathrm{~m}, 1 \mathrm{H}), 4.57(\mathrm{br} \mathrm{s}, 1 \mathrm{H}), 4.37(\mathrm{t}, \mathrm{J}=6.6 \mathrm{~Hz}, 2 \mathrm{H}), 3.84-3.73(\mathrm{~m}, 2 \mathrm{H}), 3.70-$ $3.40(\mathrm{~m}, 4 \mathrm{H}), 3.30-3.09(\mathrm{~m}, 5 \mathrm{H}), 2.95-2.85(\mathrm{~m}, 1 \mathrm{H}), 2.82-2.65(\mathrm{~m}, 2 \mathrm{H}), 2.65-2.37(\mathrm{~m}, 3 \mathrm{H})$, 2.38-2.26 (m, 1H), 2.20-2.05 (m, 2H), $1.47(\mathrm{~s}, 18 \mathrm{H}), 1.92-1.22(\mathrm{~m}, 35 \mathrm{H}), 1.16(\mathrm{~d}, \mathrm{~J}=6.2$ $\mathrm{Hz}, 3 \mathrm{H}), 0.87$ (t, J = 7.3 Hz, 3H); ${ }^{13} \mathrm{C}$ NMR (125 MHz, $\left.\mathrm{CD}_{3} \mathrm{OD}\right): \delta 181.9,176.7 / 176.3$, 167.5, 164.6, 158.5, 151.8, 133.8, 131.6, 114.6, 87.0, 80.2, 72.7, 69.2, 68.4, 68.3, 67.5, 61.0, 55.1, 53.6, 45.2, 39.7, 38.7, 30.9, 30.81, 30.78, 30.75, 30.72, 30.4, 29.04, 29.01, 27.4, 25.7, 23.9, 10.8; IR (neat) 3331, 2972, 2926, 2856, 1702, 1629, 1583, 1517, 1455, 1366, $1254,1170,1127 ;[\alpha]^{27}{ }_{589}-17.1,[\alpha]^{27}{ }_{577}-20.0,[\alpha]^{27}{ }_{546}-26.3,[\alpha]^{27}{ }_{435}-79.0(c) 0.10$, $\mathrm{MeOH}),[\alpha]^{27}{ }_{405}-99.2\left(c\right.$ 0.48, MeOH); HRMS $\left(\mathrm{ESI}^{+}\right) \mathrm{m} / \mathrm{z} 999.7110$ (999.7094 calculated for $\left.\mathrm{C}_{55} \mathrm{H}_{95} \mathrm{~N}_{6} \mathrm{O}_{10}\left[\mathrm{M}^{+}\right]\right)$. 
Amide 27. Using an identical procedure, acid 6 (16 $\mathrm{mg}, 0.023 \mathrm{mmol})$ and ent-20 ${ }^{2}$ provided amide 27 as a clear oil (19 mg, $0.018 \mathrm{mmol}, 79 \%$ yield). ${ }^{1} \mathrm{H}$ and ${ }^{13} \mathrm{C}$ NMR spectra of this compound are identical to those of 26. IR (neat) 3331, 2972, 2926, 2856, 1702, 1629, $1583,1517,1455,1366,1254,1170,1127 ;[\alpha]^{27}{ }_{589}-17.1,[\alpha]^{27}{ }_{577}-20.0,[\alpha]^{27}{ }_{546}-26.3$, $[\alpha]_{435}^{27}-79.0\left(c\right.$ 0.10, MeOH), $[\alpha]^{27}{ }_{405}-99.2$ (c 0.48, MeOH); HRMS (ESI $\left.{ }^{+}\right) \mathrm{m} / \mathrm{z} 999.7110$ (999.7094 calculated for $\mathrm{C}_{55} \mathrm{H}_{95} \mathrm{~N}_{6} \mathrm{O}_{10}\left[\mathrm{M}^{+}\right]$). 


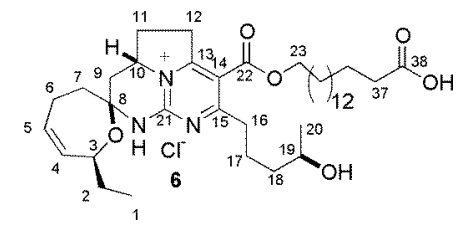

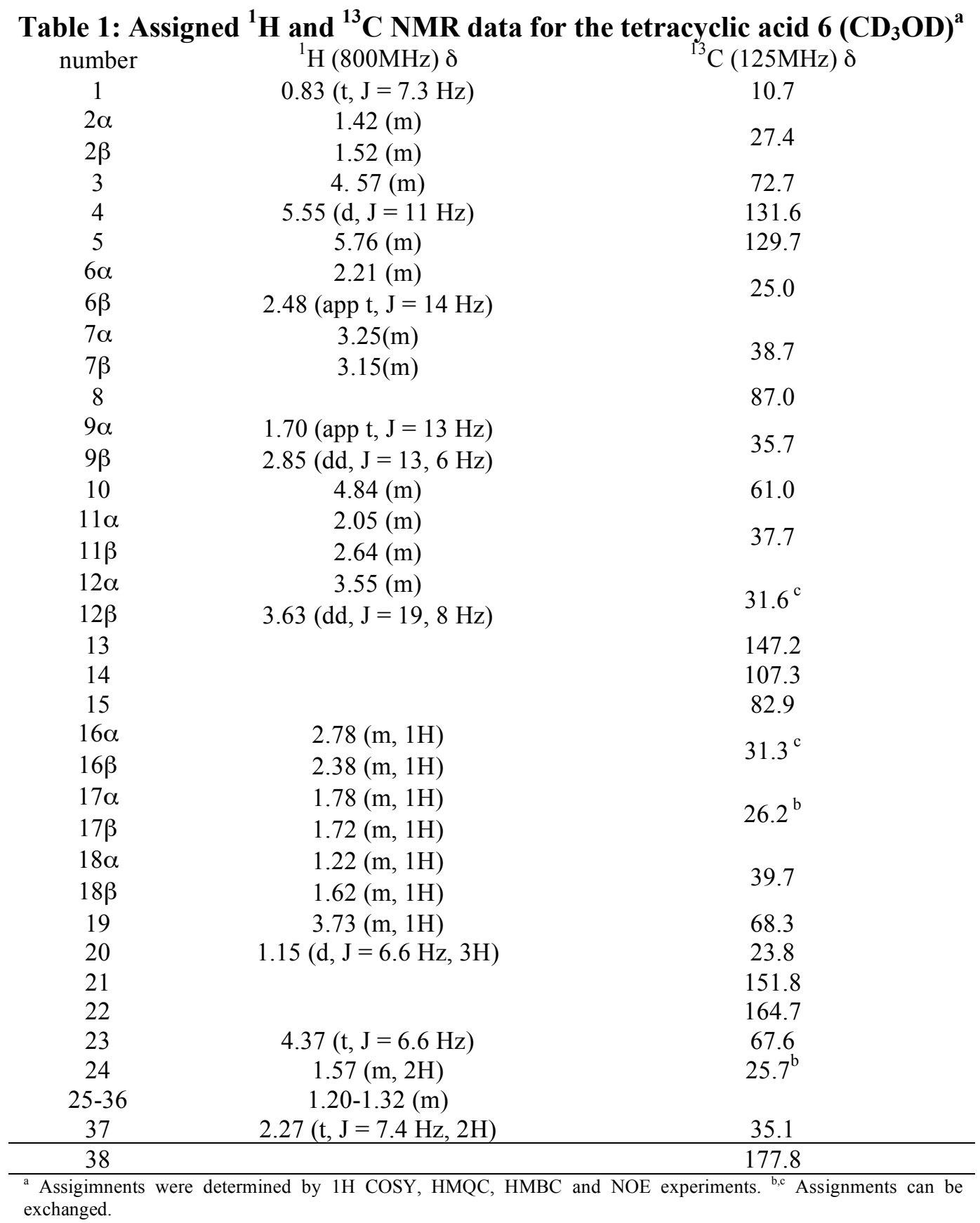



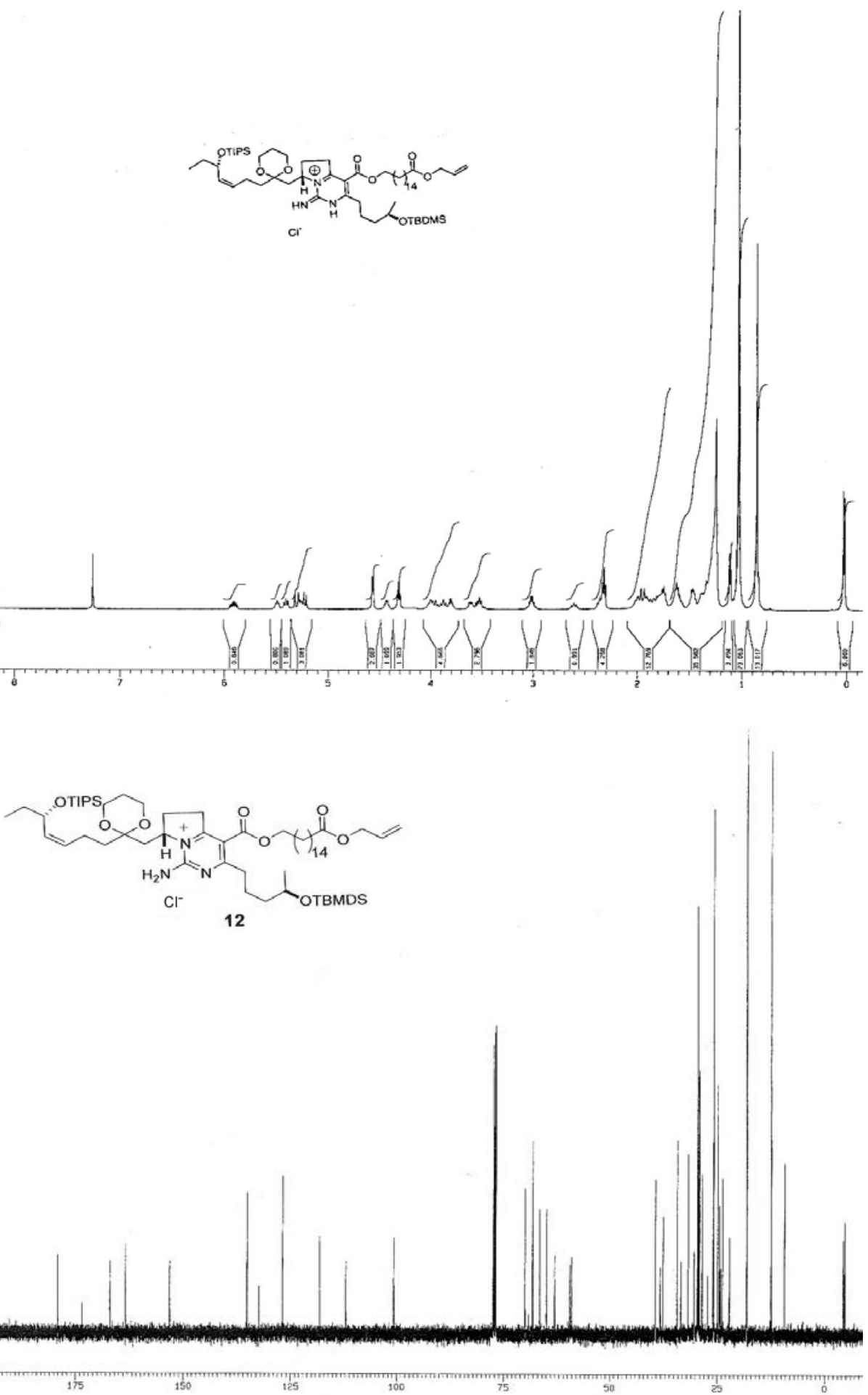


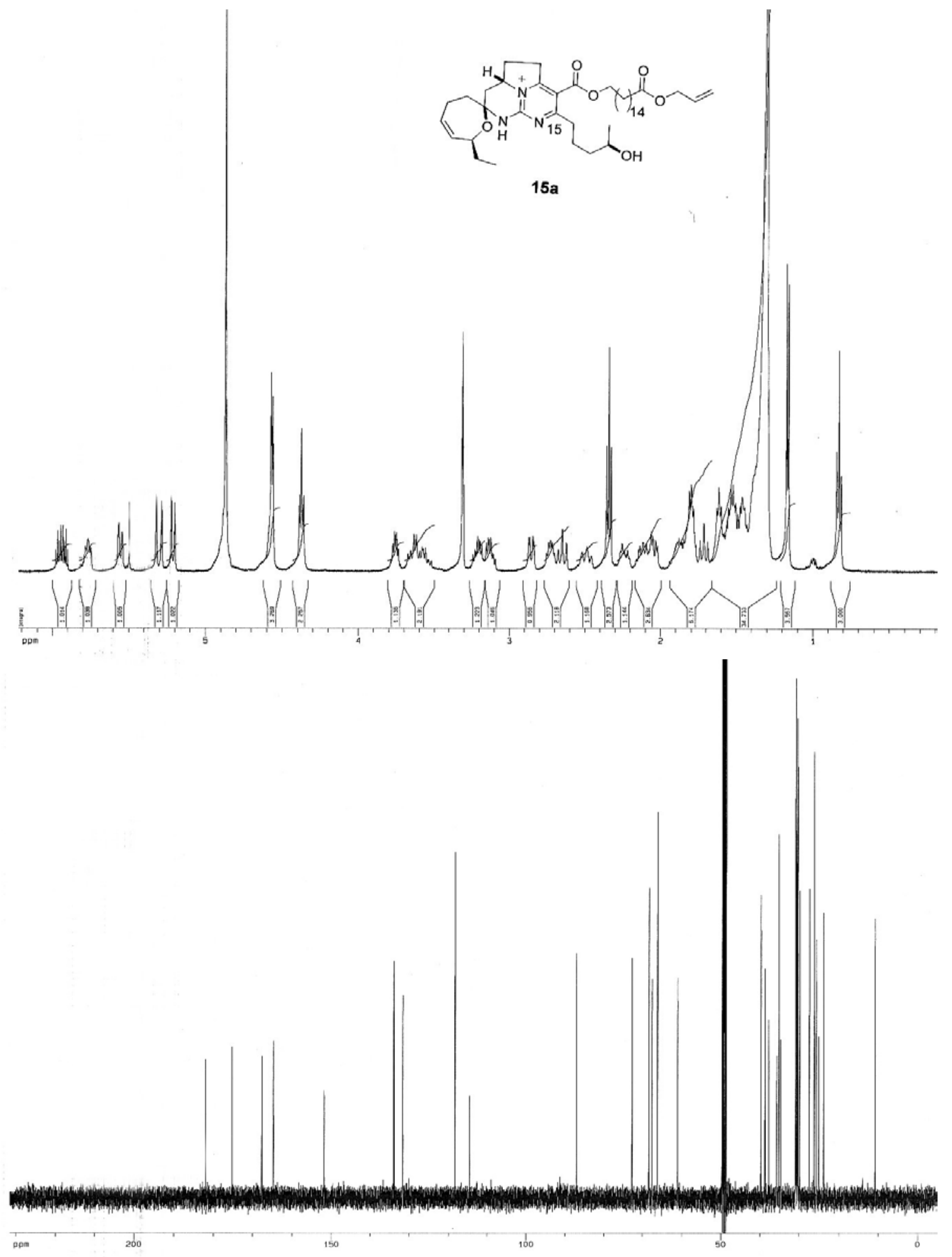




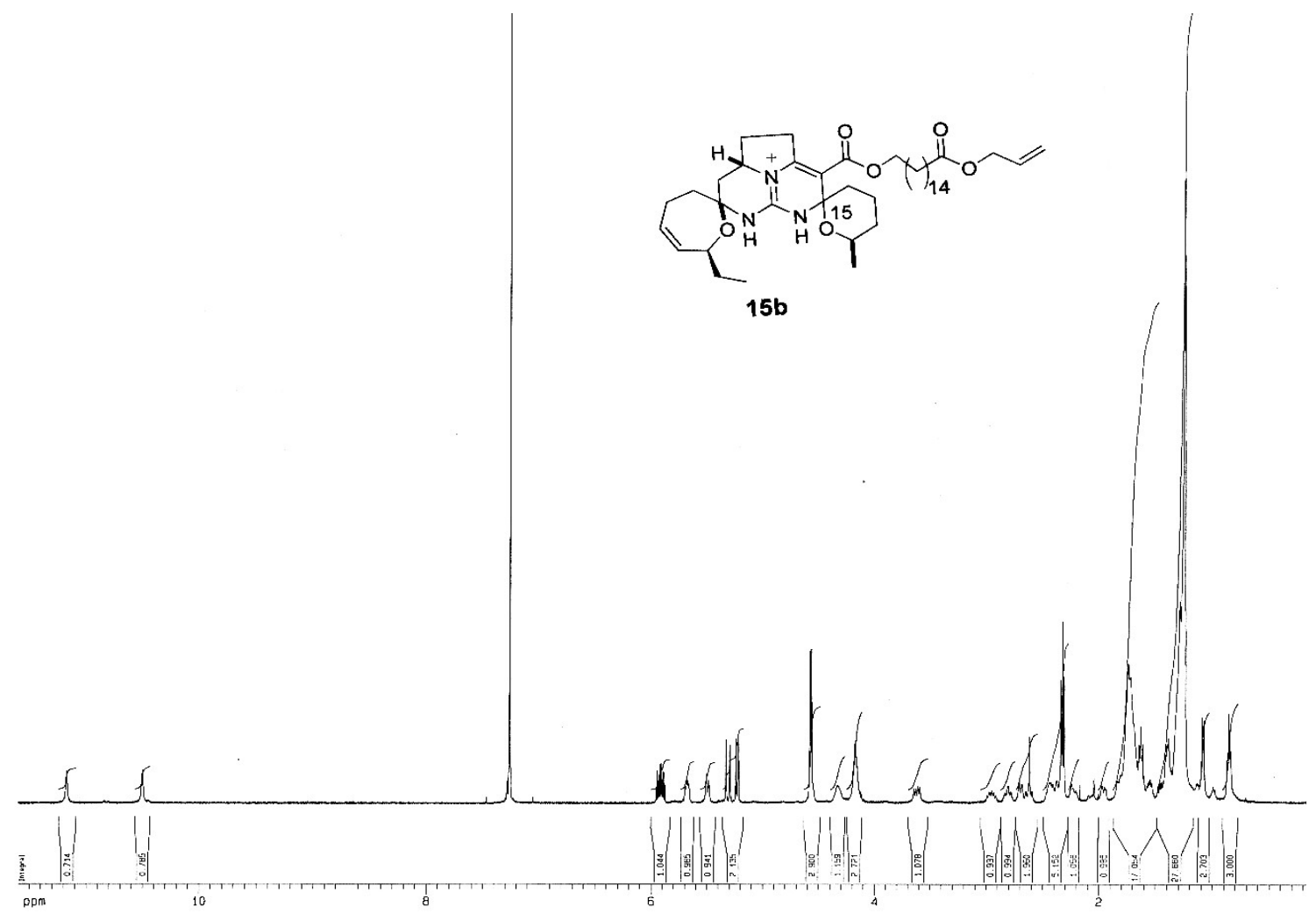




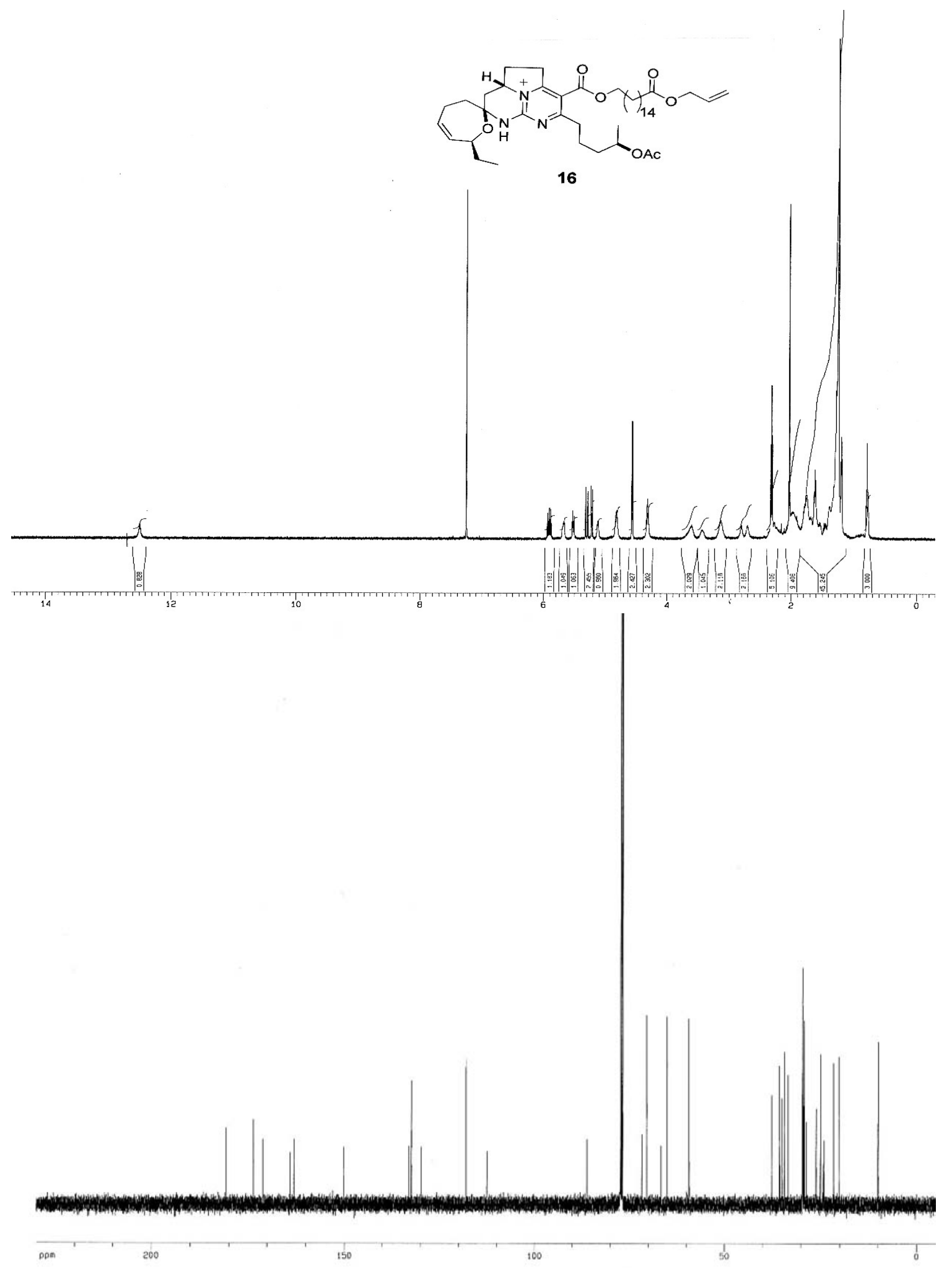




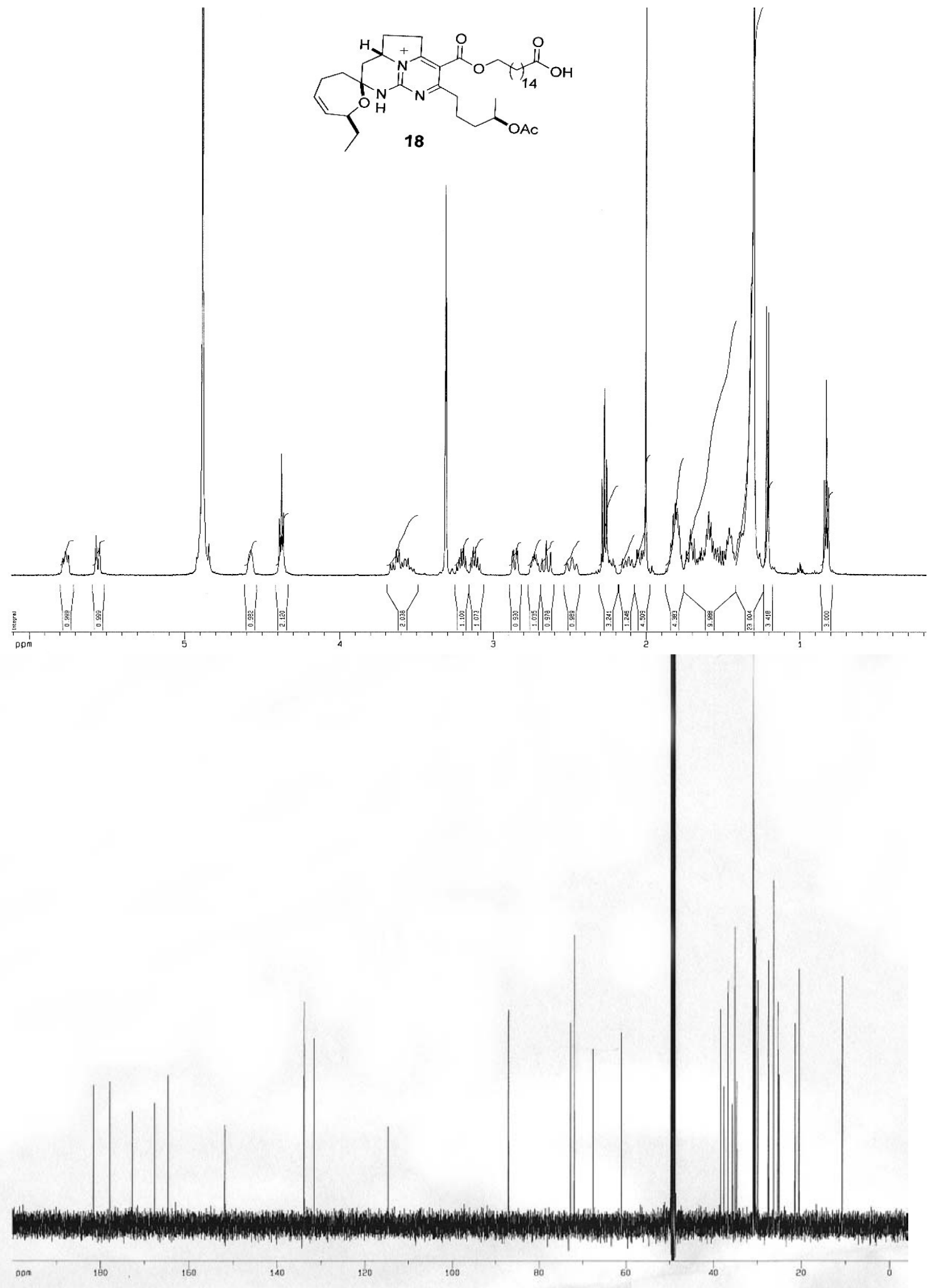




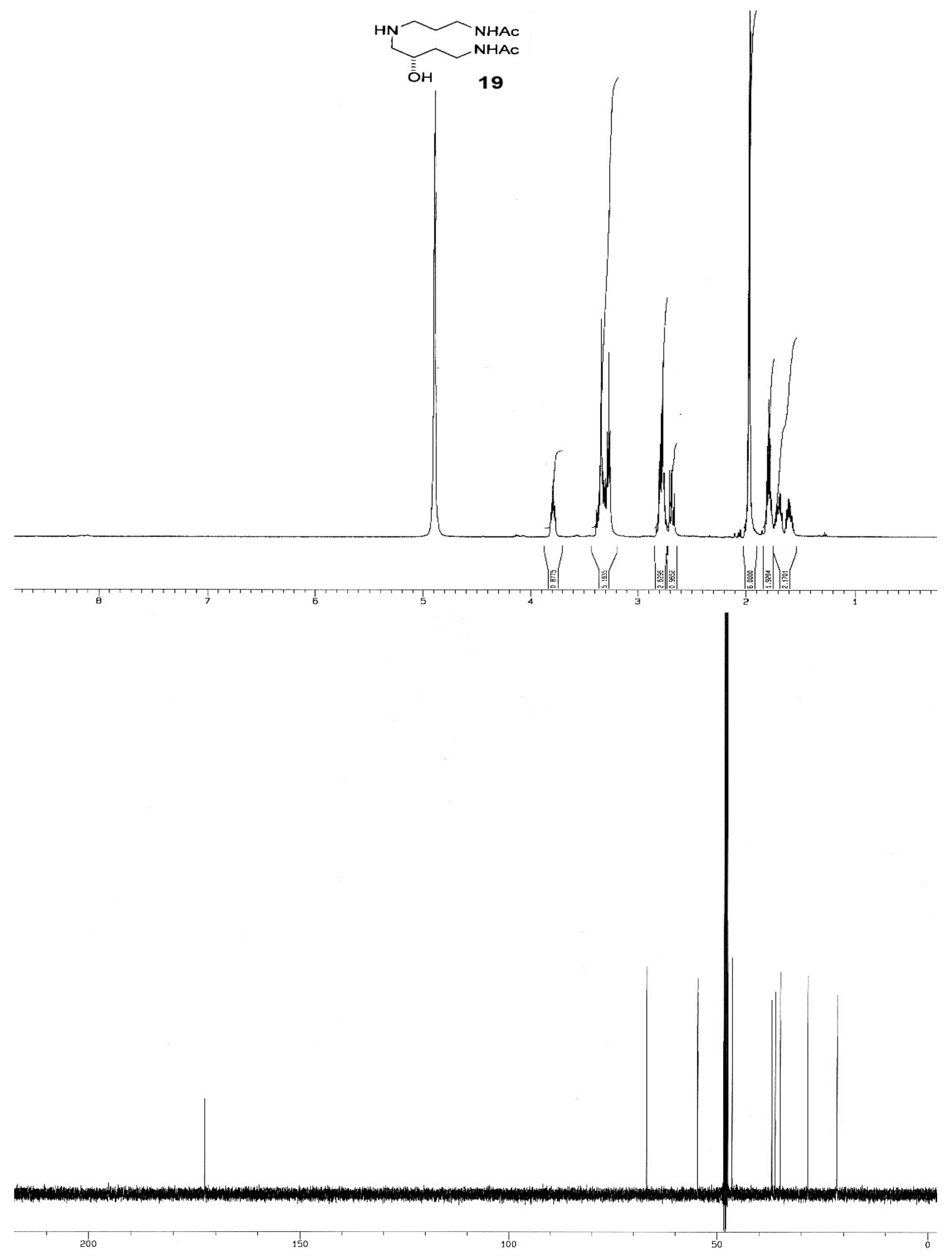



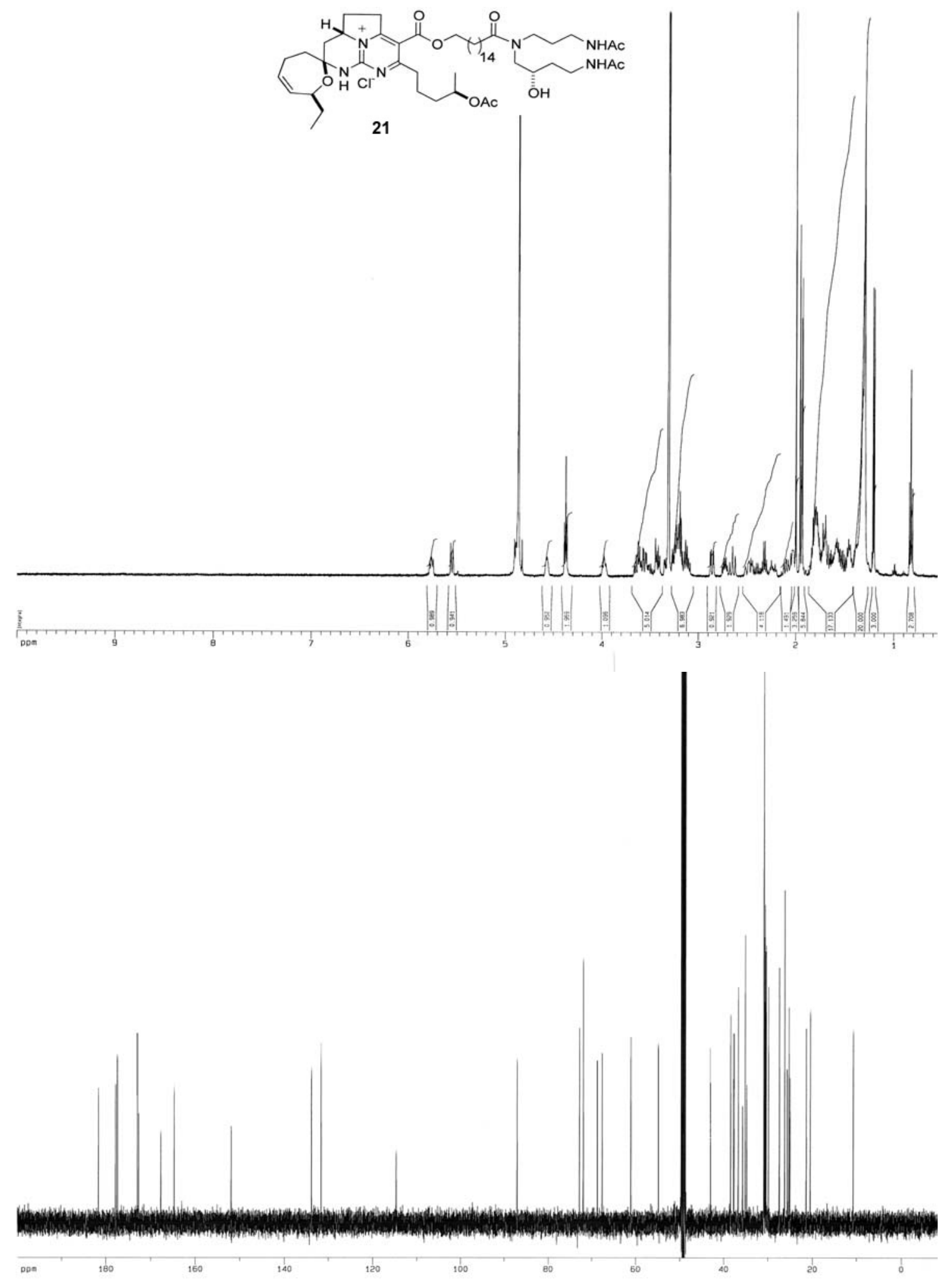


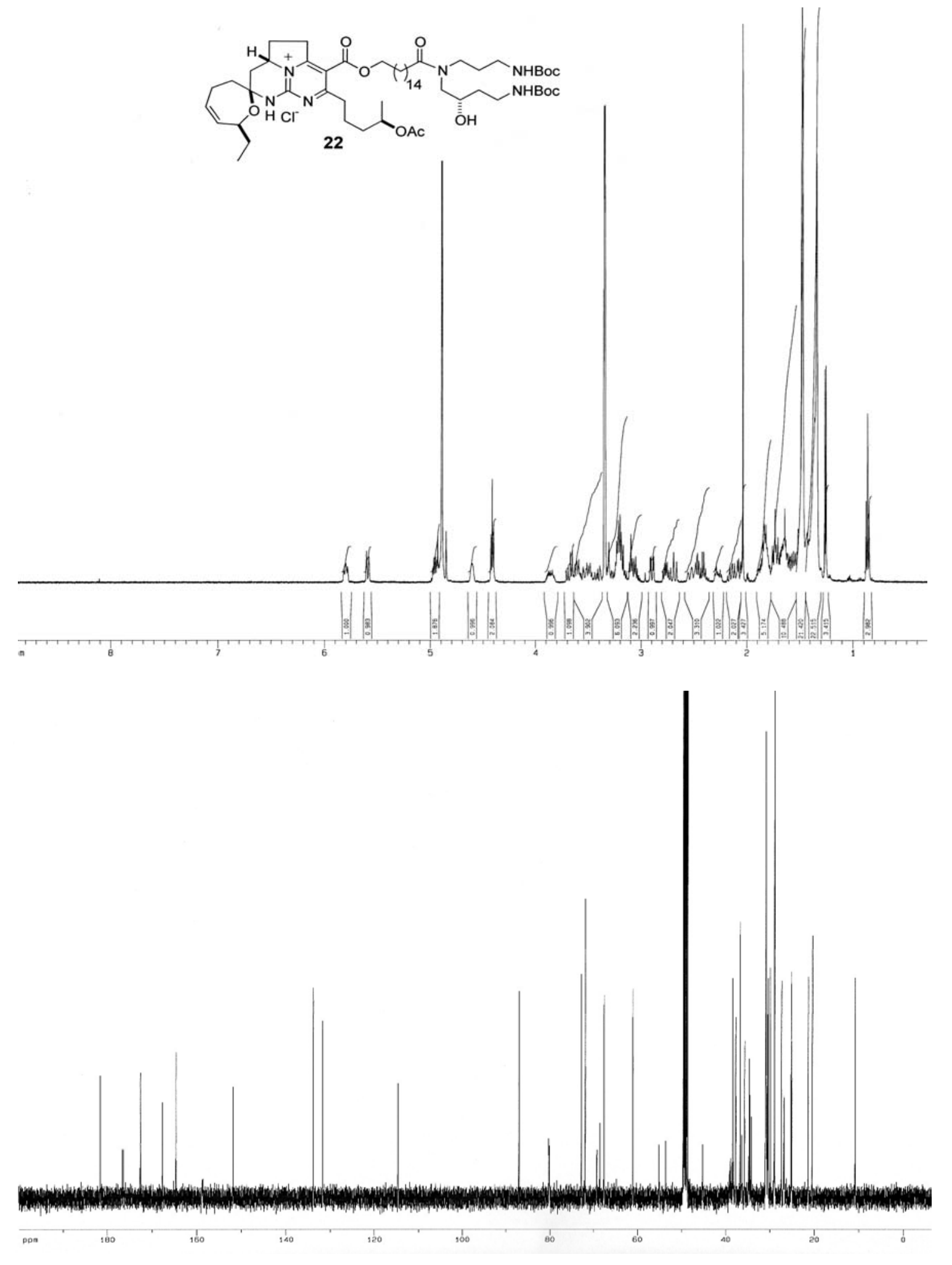



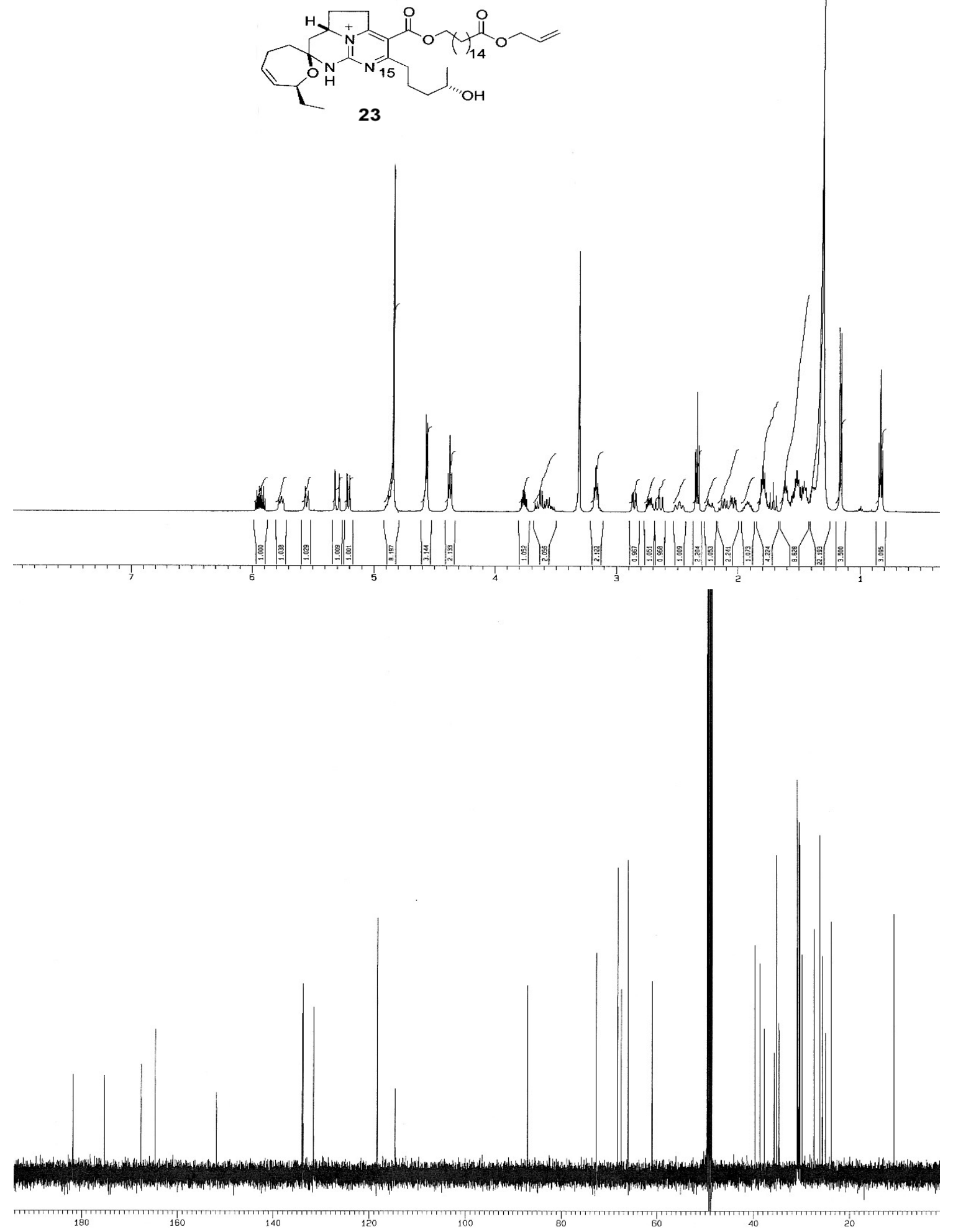


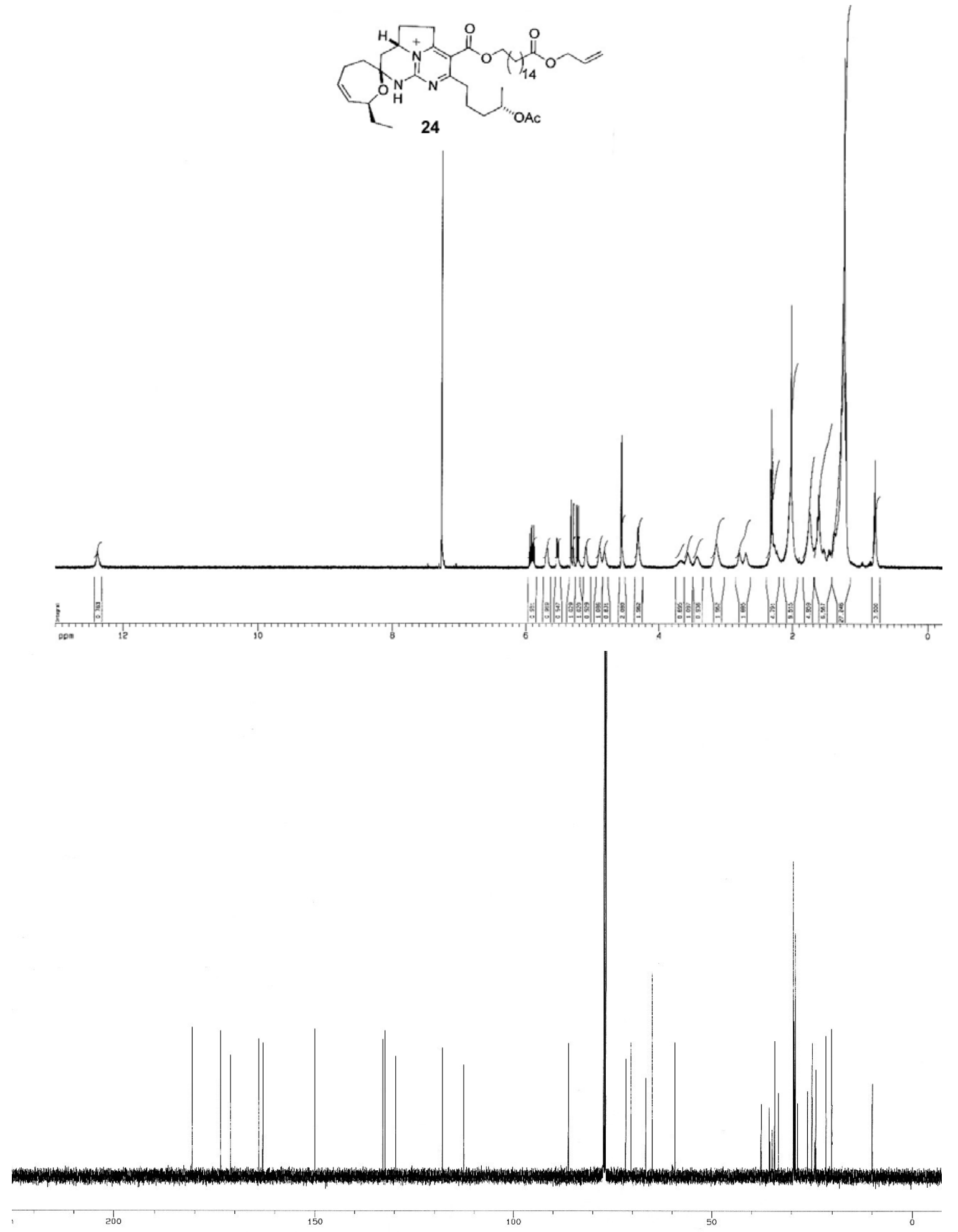



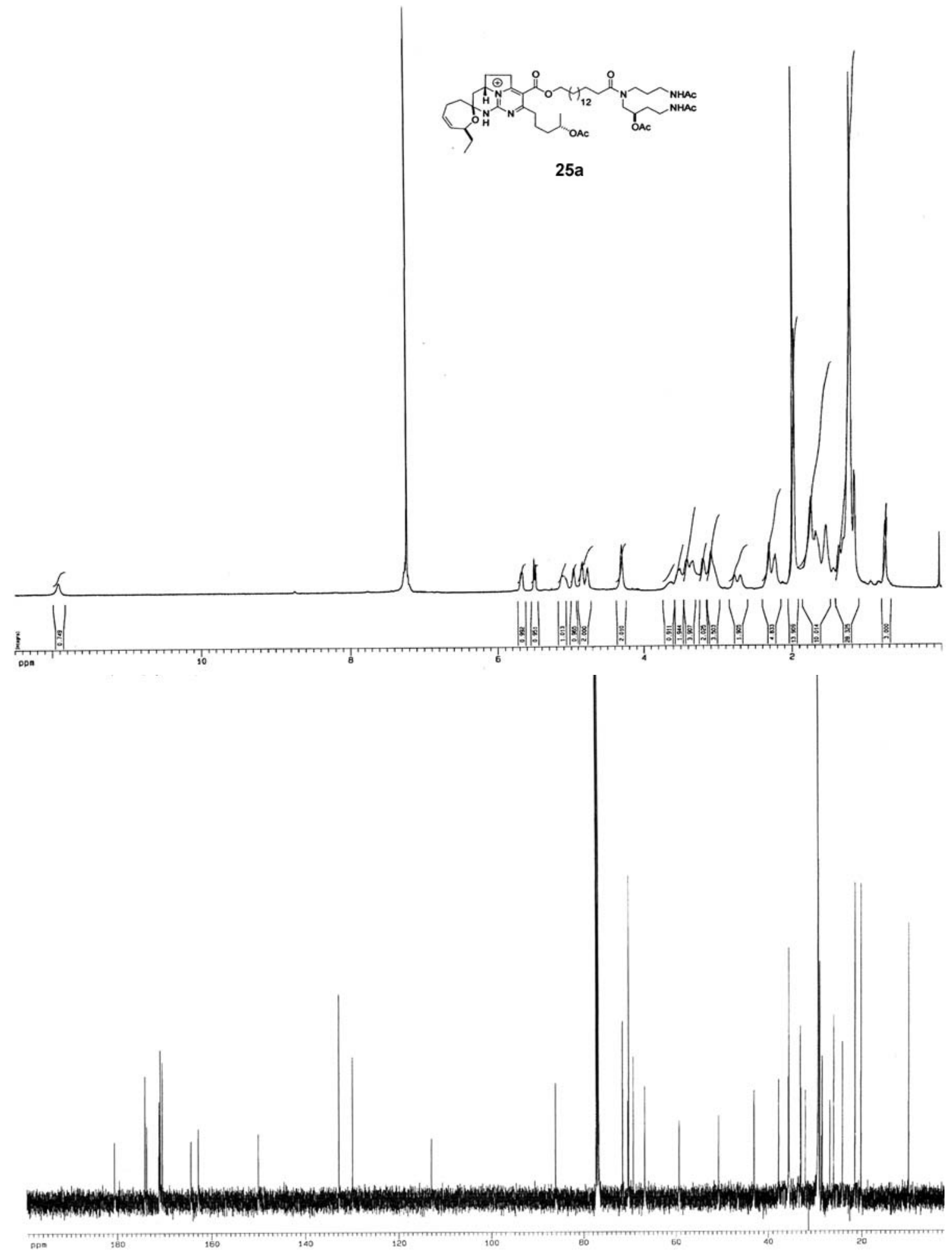


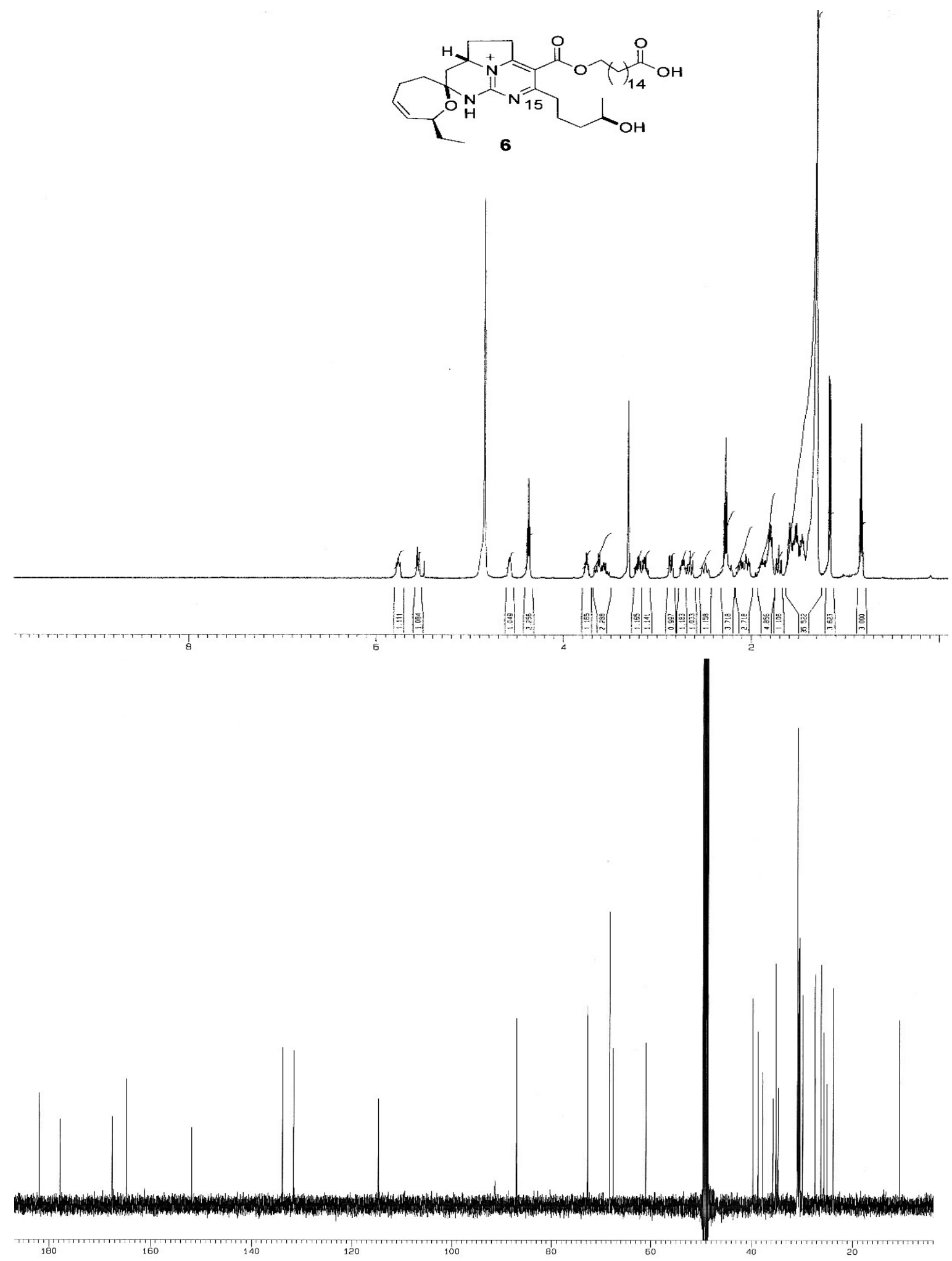

S21 


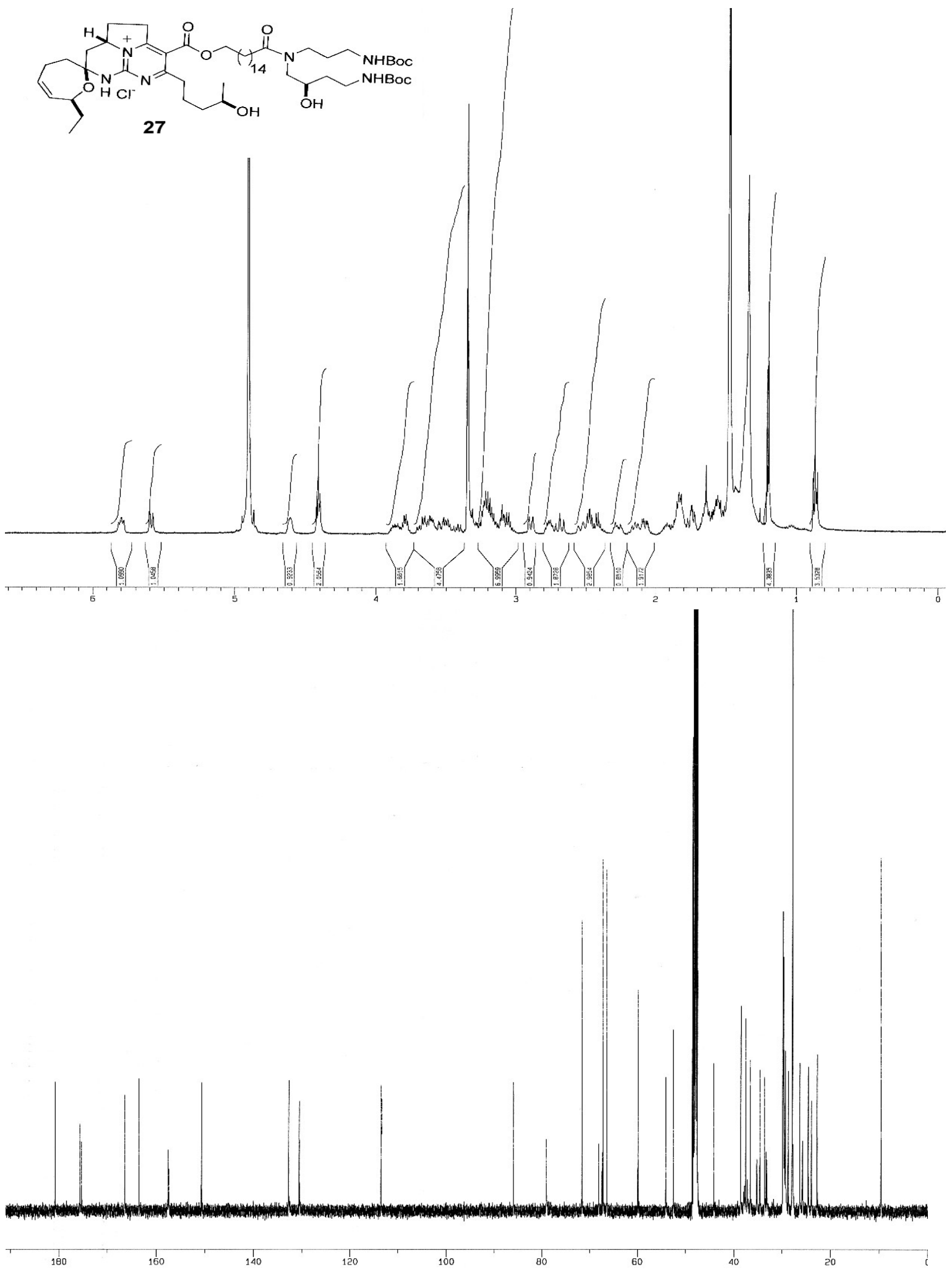

S22 


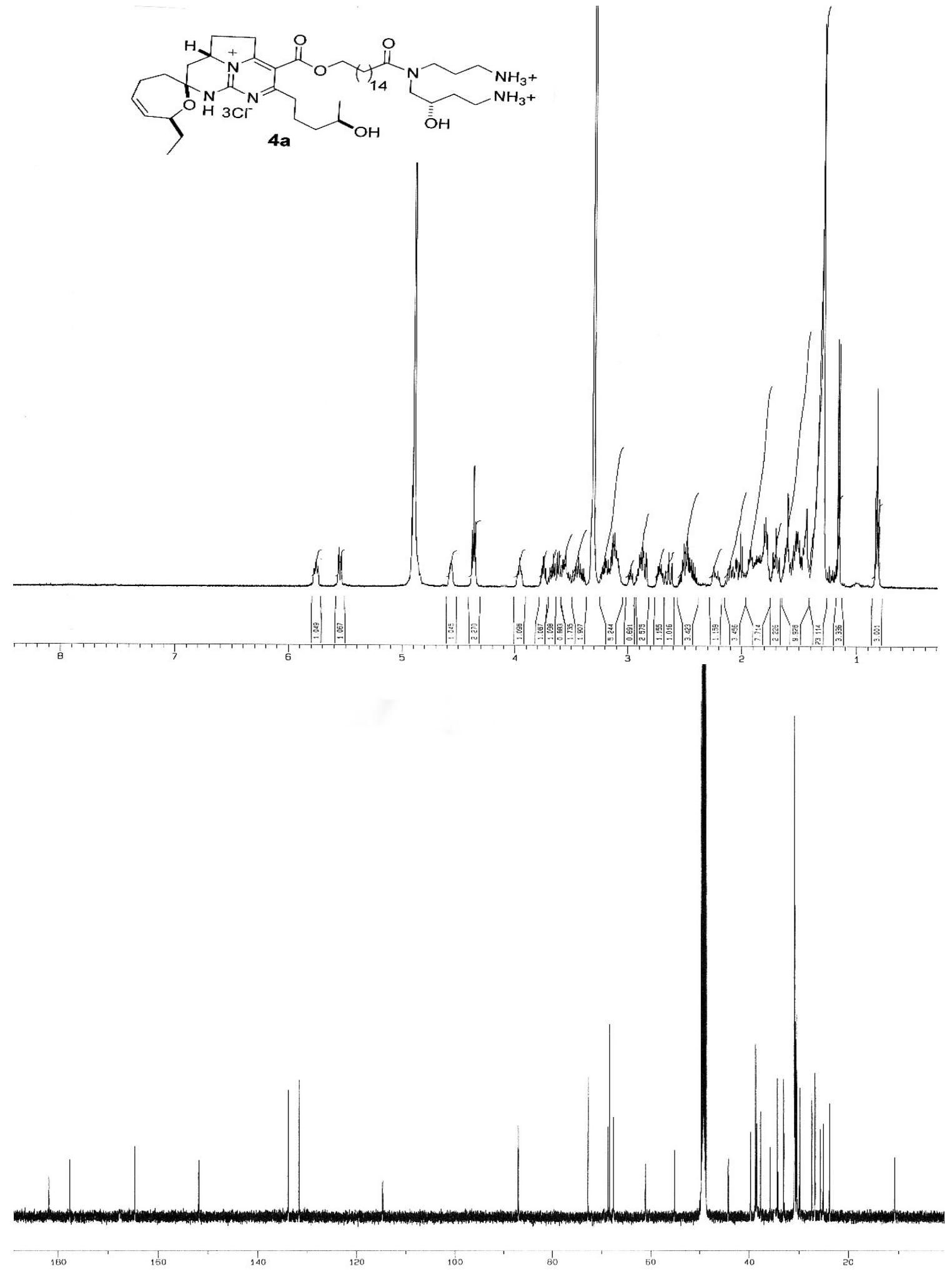

